\title{
The planetary nebulae and HII regions in NGC 6822 revisited. Clues to AGB nucleosynthesis
}

\author{
Jorge García-Rojas ${ }^{1,2}$, Miriam Peña ${ }^{3}$, Sheila Flores-Durán ${ }^{3}$, and Liliana Hernández-Martínez ${ }^{3,4}$ \\ ${ }^{1}$ Instituto de Astrofísica de Canarias, 38205 La Laguna, Tenerife, Spain \\ e-mail: jogarcia@iac.es \\ 2 Universidad de La Laguna, Dept. Astrofísica, 38206 La Laguna, Tenerife, Spain \\ 3 Instituto de Astronomía, Universidad Nacional Autónoma de México, Apdo. Postal 70-264, Méx. D. F., 04510 México, Mexico \\ ${ }^{4}$ Instituto de Ciencias Nucleares, Universidad Nacional Autónoma de México, Apdo. Postal 70-543, Méx. D. F., 04510 México, \\ Mexico
}

Received 28 August 2015 / Accepted 18 October 2015

\begin{abstract}
Aims. The chemical behaviour of an ample sample of planetary nebulae (PNe) in NGC 6822 is analysed.

Methods. Spectrophotometric data of $11 \mathrm{PNe}$ and two H II regions were obtained with the OSIRIS spectrograph attached to the Gran Telescopio Canarias. Data for other $13 \mathrm{PNe}$ and three $\mathrm{H}$ II regions were retrieved from the literature. Physical conditions and chemical abundances of $\mathrm{O}, \mathrm{N}, \mathrm{Ne}, \mathrm{Ar}$, and $\mathrm{S}$ were derived in a consistent way for $19 \mathrm{PNe}$ and $4 \mathrm{H}$ II regions.

Results. Abundances in the PNe sample are widely distributed showing $12+\log (\mathrm{O} / \mathrm{H})$ from 7.4 to 8.2 and $12+\log (\mathrm{Ar} / \mathrm{H})$ from 4.97 to 5.80. Two groups of PNe can be differentiated: one old with low metallicity $(12+\log (\mathrm{O} / \mathrm{H})<8.0$ and $12+\log (\mathrm{Ar} / \mathrm{H})<5.7)$ and another younger one with metallicities similar to the values for $\mathrm{H}$ II regions. The old objects are distributed in a larger volume than the young ones. An important fraction of PNe (over 30\%) was found to be highly N-rich (Peimbert Type I PNe). Such PNe occur at any metallicity. In addition, about $60 \%$ of the sample presents high ionization $\left(\mathrm{He}^{++} / \mathrm{He} \geq 0.1\right)$, possessing a central star with effective temperature higher than $100000 \mathrm{~K}$. Possible biases in the sample are discussed. From comparison with stellar evolution models by Karakas (2010) and Fishlock et al. (2014) of the observed N/O abundance ratios, our PNe should have had initial masses that are lower than $4 M_{\odot}$, although if the comparison is made with $\mathrm{Ne}$ vs. $\mathrm{O}$ abundances, the initial masses should have been lower than $2 M_{\odot}$. It appears that these models of stars of $2-3 M_{\odot}$ are producing too much ${ }^{22} \mathrm{Ne}$ in the stellar surface at the end of the AGB. On the other hand, the comparison with another set of stellar evolution models with a different treatment of convection and on the assumptions about the overshoot of the convective core during the core H-burning phase, provided there is reasonable agreement between the observed and predicted $\mathrm{N} / \mathrm{O}$ and $\mathrm{Ne} / \mathrm{H}$ ratios if initial masses of more massive stars are about $4 M_{\odot}$.
\end{abstract}

Key words. ISM: abundances - HII regions - planetary nebulae: general - galaxies: abundances - galaxies: dwarf stars: AGB and post-AGB

\section{Introduction}

The barred irregular galaxy NGC 6822 (IBs(m), also known as DDO209), is one of the most interesting galaxies in the Local Group. Half way between the Milky Way and Andromeda, (Gieren et al. 2006, reported a distance modulus of $23.31 \pm$ 0.02 ), it presents several very interesting characteristics that have made it the subject of intense studies.

NGC 6822 contains different populations with different spatial distributions and kinematics. Its optical structure is dominated by a bar that is $8^{\prime}$ long (about $1.1 \mathrm{kpc}$ ), oriented almost N-S, and it contains a huge disk of $\mathrm{H}$ I of about $6 \times 13 \mathrm{kpc}$ at a position angle (PA) of $130^{\circ}$ centred on the optical centre (de Blok \& Walter 2000; Weldrake et al. 2003). In addition, a huge spheroidal stellar distribution with its long axis at PA of $64.5^{\circ}$ (almost perpendicular to the $\mathrm{HI}$ disk), composed mainly of intermediate-age stars, has been found (Letarte et al. 2002; Battinelli et al. 2006). Interestingly, the H I disk and the stellar spheroid do not share the same kinematics. The stellar spheroid has a systemic radial velocity $v_{\mathrm{sys}}=-32.9 \mathrm{~km} \mathrm{~s}^{-1}$, and it rotates around its minor axis with radial velocities extending from 20 to $-70 \mathrm{~km} \mathrm{~s}^{-1}$ (Demers et al. 2006), while the H I disk shows a systemic velocity $v_{\mathrm{sys}}=-57 \mathrm{~km} \mathrm{~s}^{-1}$ and rotates around an axis almost orthogonal to the main axis of the spheroid, with velocities extending from $-51 \mathrm{~km} \mathrm{~s}^{-1}$ to $100 \mathrm{~km} \mathrm{~s}^{-1}$ (de Blok \& Walter 2006). A recent study of several stellar clusters in NGC 6822 (Hwang et al. 2014) reveals that the clusters seem to show completely different kinematics with a mean radial velocity of $-88 \pm 22.7 \mathrm{~km} \mathrm{~s}^{-1}$.

In recent years we have been analysing the PN population in this galaxy and other galaxies and its relation with other objects (e.g., Peña et al. 2007 for NGC 3109; Hernández-Martínez et al. 2009, 2011, for NGC 6822; Stasińska et al. 2013 for NGC 300).

Chemically, NGC 6822 is a metal-poor galaxy similar to the SMC, with an interstellar medium (ISM) abundance of about $0.2 Z_{\odot}$ (Richer \& McCall 2007). Hernández-Martínez \& Peña (2009) discovered 26 PN candidates in NGC 6822, and Hernández-Martínez et al. (2009) analysed the chemical abundances of a limited sample of them (11 objects), showing that the studied planetary nebulae (PNe) belong to two groups of different ages (1-3 Gyr and 3-9 Gyr) and different abundances, the first one with a composition similar to the present ISM and 
the older one with abundances a factor of 2 lower. By studying abundances in H II regions, they also found that the ISM located in the $2 \mathrm{kpc}$ around the centre is chemically homogeneous, showing $12+\log (\mathrm{O} / \mathrm{H})=8.06 \pm 0.04$.

Carigi et al. (2006) constructed chemical evolution models that fit the photometric properties of NGC 6822 computed by Wyder $(2001,2003)$ and derived a robust star formation history (SFH) for this galaxy. Detailed galactic chemical evolution models were computed by Hernández-Martínez et al. (2009, 2011) using PNe and $\mathrm{H}$ II region abundances as constraints with no conclusive results. One of the two best models reproduces the abundances of $\mathrm{O}, \mathrm{Ne}, \mathrm{S}, \mathrm{Ar}$, and $\mathrm{C}$ derived from collisionally excitation lines (CELs) if the most massive star in the initial mass function has a mass $M_{\text {up }}=40 M_{\odot}$. The other model reproduces the abundances obtained from recombination lines (RLs, which are about a factor of 2 higher), if a $M_{\text {up }}=80 M_{\odot}$ is assumed. This is a direct effect of the abundance discrepancy problem, a still unresolved problem for photoionized nebulae, which consists in the well known fact that abundances obtained from RLs are systematically larger than those obtained from CELs of the same ion (e.g., García-Rojas \& Esteban 2007; Liu 2012; Esteban et al. 2009). This effect is generally parametrized by the abundance discrepancy factor (ADF), which is defined as the ratio between abundances obtained from RLs and CELs. The ADF is usually between 1.5 and 3, but in PNe it has a significant tail that extends to much higher values, up to 120 (Corradi et al. 2015a). By analysing high-resolution spectra, Flores-Durán et al. (2014) demonstrate that the kinematics of PNe is closer to the one shown by the stellar spheroid than to the $\mathrm{H}$ I disk, thus PNe would belong to the intermediate age population.

In this work we revisit the PN population in order to analyse the chemical behaviour of a larger sample of objects. New data were obtained from observations with the Gran Telescopio Canarias (GTC). In Sect. 2 we present the observations and data reduction. Section 3 is devoted to discussing the true nature of the sample. In Sect. 4 the physical conditions (electron temperatures and densities) and ionic abundances of a wide sample of objects are calculated. In Sect. 5 the total abundances are derived and discussed in comparison with stellar evolution models from the literature. Our results are presented in Sect. 6, and the final summary is found in Sect. 7.

\section{Observations and data reduction}

Long-slit spectroscopy was performed with the OSIRIS spectrograph attached to the GTC, which was in service mode during $2014^{1}$. Three exposures of $900 \mathrm{~s}$ each were obtained for each slit position. A binning of $2 \times 2$ was used. Grism R1000B (IR_G3.2) was employed to cover from 3630 to $7500 \AA$ with an effective resolution of $\sim 1000$ at $7510 \AA$. This wavelength range and resolution are well suited to detecting the main plasma diagnostic line ratios, which are useful for electron temperature and electron density determinations in the nebula, which are crucial for an accurate ionic abundance determination.

The slit size was 6.8 arcmin long by 1.5 arcsec wide. Slits were oriented in a mode where two or more objects were observed simultaneously in each observing block (OB) to save exposure time. The log of observations is presented in Table 1, where the slit position angle and observed objects in each $\mathrm{OB}$ are

\footnotetext{
1 GTC programme number is GTC5-14AIACMEX, and $6 \mathrm{~h}$ of observing time were awarded. The observing time was divided in 6 observing blocks (OB), one hour each, of which 45 min were used for scientific observations.
}

Table 1. Log of observations with GTC.

\begin{tabular}{lllll}
\hline \hline Block & Date & Slit PA $^{a}$ & A.M. & Objects $^{b}$, night condition \\
\hline OB1 & $2014-06-02$ & $-26.4^{\circ}$ & 1.387 & PN 2, PN 8, dark clear \\
OB2 & $2014-06-03$ & $+54.4^{\circ}$ & 1.397 & PN 25, PN 26, dark spectr. \\
OB3 & $2014-06-23$ & $-10.3^{\circ}$ & 1.502 & PN 23, PN 24, dark clear \\
OB4 & $2014-06-24$ & $+1.3^{\circ}$ & 1.380 & PN 11, PN 15, dark clear \\
OB5 & $2014-06-27$ & $-0.35^{\circ}$ & 1.405 & PN 9, PN 22, dark phot. \\
OB6 & $2014-06-27$ & $+3.8^{\circ}$ & 1.389 & PN 13, H II, H III, dark phot. \\
\hline
\end{tabular}

Notes. Exposure time was always $3 \times 900 \mathrm{~s}$, for each OB. ${ }^{(a)}$ Slit size was always 6.8 arcmin $\times 1.5$ arcsec. ${ }^{(b)}$ PN names as in Hernández-Martínez \& Peña (2009).

included. Because PNe are faint objects, most of the time a blind offset from a nearby field star was needed to place the objects in the slit. In total we observed $11 \mathrm{PN}$ candidates and two faint $\mathrm{H}$ II regions. The effect of atmospheric differential refraction was minimized by observing NGC 6822 close to culmination, which translates into slit orientations that are very close to the parallactic angle (differences less than $20-30^{\circ}$ ). This was not the case for OB2 where slit position was more than $60^{\circ}$ away from parallactic angle; however, spectra of PN 25 and PN 26 were not useful for our purposes, probably owing to large slit losses.

Observed 2D spectra were bias-subtracted and flat-fielded. Afterwards the spectra were extracted, and a careful sky subtraction was made, especially in the red zone where many sky lines appear. The extraction window was chosen in such a way that all the PN nebular emission was included. For H II regions, which are more extended, the extraction window was $3^{\prime \prime}$ and generally it does not include the whole object. The standard star Ross 640 was used for flux calibration. $\mathrm{Ne}$ and $\mathrm{Hg}$-Ar lamps were observed in daytime for spectral calibration. Calibrated spectra of $\mathrm{PNe}$ and two H II regions are shown in Fig. A.1.

Dereddened nebular line fluxes are presented in Table A.1, which for each object, includes the logarithmic reddening correction, $\mathrm{c}(\mathrm{H} \beta)$, and the $\mathrm{H} \beta$ observed flux. $\mathrm{c}(\mathrm{H} \beta)$ was derived from the Balmer ratio $\mathrm{H} \alpha / \mathrm{H} \beta$, by assuming Case B (Storey \& Hummer 1995). No underlying absorption was considered to correct $\mathrm{H} \alpha$ and $\mathrm{H} \beta$ emission of $\mathrm{H}$ II regions since these lines have a very large equivalent width. In the case of PNe, the central stars are very faint, and no underlying absorption is expected to affect the nebular lines. An additional object observed, but not included in Table A.1, is PN 26 for which only $\mathrm{H} \alpha$ was detected with a flux of 7.93E-17 erg cm $\mathrm{es}^{-2}$.

Additional spectroscopic data were obtained from the literature. The line fluxes reported by Hernández-Martínez et al. (2009) from VLT-FORS2 observations for PN 4, PN 5, PN 6, PN 7, PN 10, PN 12, PN 14, PN 16, PN 18, PN 19, PN 21, and H II 15; those by Richer \& McCall (2007), from observations with the Canada-France-Hawaii (CFH) telescope and the MultiObject Spectrograph MOS, for PN 17 and PN 20, and data published by Peimbert et al. (2005) for the H II regions $\mathrm{H} \mathrm{V}$ and $\mathrm{HX}$ : all were retrieved and physical conditions and chemical abundances recalculated, following the same procedure as for our GTC data to have a consistent data set of abundances.

\section{The true nature of objects observed with GTC}

At the distance of NGC 6822, PN candidates can be easily mixed up with very compact $\mathrm{H}$ II regions, therefore spectroscopic data are needed to segregate both types of objects. In our sample of $11 \mathrm{PN}$ candidates observed with GTC, we found that the He II $\lambda 4686$ emission line is present (and measurable) in the 

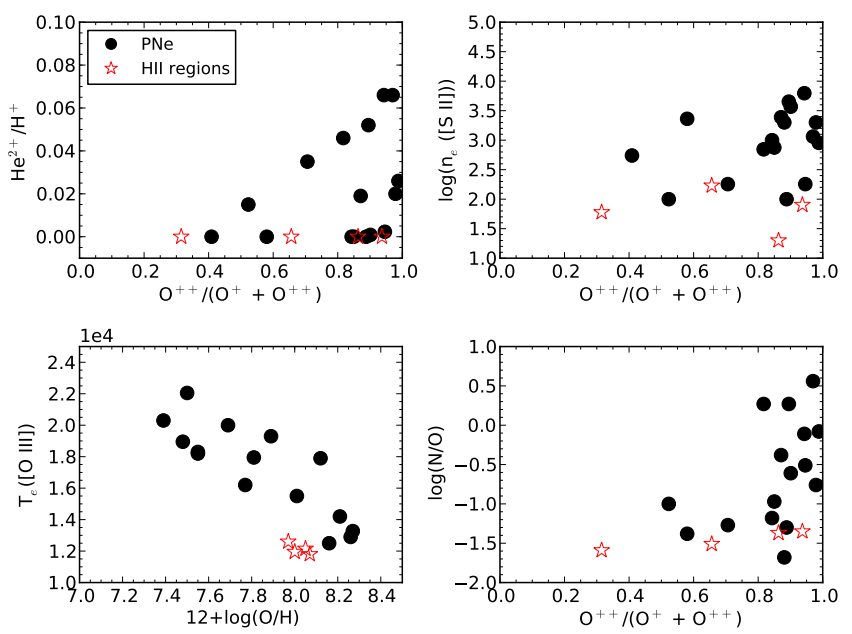

Fig. 2. Diagrams illustrating observed properties that allow differentiatin between PNe and H II regions in NGC 6822.

objects named PN 8, PN 23, and PN 24, therefore their PN nature is confirmed since it is not expected that $\mathrm{H}$ II regions emit such a high ionization line. In addition, the [O III] $\lambda 5007 / \mathrm{H} \beta$ line ratio is larger than 3 in the objects PN 2, PN 11, and PN 13, also confirming their PN nature. The objects PN 9, PN 15, PN 22, PN 25, and PN 26 are very faint with observed $\mathrm{F}(\mathrm{H} \beta)$ lower than about $2 \times 10^{-16} \mathrm{erg} \mathrm{cm}^{-2} \mathrm{~s}^{-1}$, and no (or very faint) [O III] $\lambda 5007$ or He II $\lambda 4686$ emission were detected, thus their PN nature is still unconfirmed, although the faintness of $\mathrm{H} \beta$, the stellar appareance, and the lack of a detectable central star support their PN nature. In particular, we want to point out the case of PN 25, for which the [N II] $\lambda \lambda 6548,6583$ lines are stronger than $\mathrm{H} \alpha$ (see Fig. A.1), which makes it a good candidate for a Peimbert Type I PN. As mentioned above, only $\mathrm{H} \alpha$ was detected for PN 26.

By considering the 11 objects confirmed as PNe by Hernández-Martínez et al. (2009), the two observed by Richer $\&$ McCall (2007), and the six objects observed with GTC and confirmed as PNe in this work, we find that 19 of the 26 PN candidates reported by Hernández-Martínez \& Peña (2009) are true $\mathrm{PNe}$, and the others are highly probable $\mathrm{PNe}$, thus indicating that the criteria for selecting PN candidates in external galaxies proposed by these authors are very good. However, as we see in the discussion, these criteria, which were designed to mainly select high-luminosity ([O III] $\lambda 5007$ ) PNe (useful for building the planetary nebula luminosity function, PNLF), lead to ignore a number of $\mathrm{PNe}$, in particular those with low excitation and low [O III] $\lambda 5007$ flux. Our sample therefore shows some bias that will be discussed later. In Sect. 5 we present Fig. 2, which shows some diagrams that are useful for distinguishing between $\mathrm{PNe}$ and compact $\mathrm{H}$ II regions.

\section{Physical conditions and ionic chemical abundances}

All the available diagnostic line ratios were used to derive the physical conditions. Electron temperatures were calculated from [O III] $\lambda \lambda 4363 / 5007$ and [N II] $\lambda \lambda 5755 / 6583$ intensity ratios, while densities were derived from [S II] $\lambda \lambda 6717 / 6731$ and [ArIV] $\lambda \lambda 4711 / 4740$ intensity ratios. For $\mathrm{HV}$ and $\mathrm{HX}$, we can also compute electron temperatures from the [O II] $\lambda \lambda 3727 / 7325$ intensity ratio and electron densities from the [O II] $\lambda \lambda 3726 / 3729$ and the [Cl III] $\lambda \lambda 5517 / 5537$ line ratios; the last ratio was also measured for H II 15 (see Table 2 of
Table 2. Atomic data set used for collisionally excited lines.

\begin{tabular}{lcc}
\hline \hline Ion & $\begin{array}{c}\text { Transition } \\
\text { probabilities }\end{array}$ & $\begin{array}{c}\text { Collisional } \\
\text { strengths }\end{array}$ \\
\hline $\mathrm{N}^{+}$ & Froese Fischer \& Tachiev (2004) & Tayal (2011) \\
$\mathrm{O}^{+}$ & Froese Fischer \& Tachiev (2004) & Kisielius et al. (2009) \\
$\mathrm{O}^{2+}$ & Froese Fischer \& Tachiev (2004) & Storey et al. (2014) \\
& Storey \& Zeippen (2000) & \\
$\mathrm{Ne}^{2+}$ & Galavís et al. (1997) & McLaughlin \& Bell (2000) \\
$\mathrm{Ne}^{4+}$ & Galavís et al. (1997) & Dance et al. (2013) \\
& Bhatia \& Doschek (1993) & \\
$\mathrm{S}^{+}$ & Podobedova et al. (2009) & Tayal \& Zatsarinny (2010) \\
$\mathrm{S}^{2+}$ & Podobedova et al. (2009) & Tayal \& Gupta (1999) \\
$\mathrm{Cl}^{2+}$ & Mendoza (1983) & Butler \& Zeippen (1989) \\
$\mathrm{Ar}^{2+}$ & Mendoza (1983) & Galavís et al. (1995) \\
& Kaufman \& Sugar (1986) & \\
$\mathrm{Ar}^{3+}$ & Mendoza \& Zeippen (1982) & Ramsbottom \& bell (1997) \\
$\mathrm{Ar}^{4+}$ & Mendoza \& Zeippen (1982) & Galavís et al. (1995) \\
& Kaufman \& Sugar (1986) & \\
& LaJohn \& Luke (1993) &
\end{tabular}

Table 3. Lines used for ionic abundance determinations.

\begin{tabular}{ll}
\hline \hline Ion & Line \\
\hline $\mathrm{N}^{+}$ & {$[\mathrm{N} \mathrm{II}] \lambda \lambda 6548,6584$} \\
$\mathrm{O}^{+}$ & {$[\mathrm{O}$ II $] \lambda \lambda 3726+29,7320+30$} \\
$\mathrm{O}^{++}$ & {$[\mathrm{O} \mathrm{III}] \lambda \lambda 4959,5007$} \\
$\mathrm{Ne}^{++}$ & {$[\mathrm{Ne}$ III] $\lambda \lambda 3868$} \\
$\mathrm{Ne}^{+4}$ & {$[\mathrm{Ne}$ V] $\lambda 3425$} \\
$\mathrm{S}^{+}$ & {$[\mathrm{S} \mathrm{II}] \lambda \lambda 6717+31,4068+76$} \\
$\mathrm{~S}^{++}$ & {$[\mathrm{S} \mathrm{III}] \lambda 6311$} \\
$\mathrm{Cl}^{++a}$ & {$[\mathrm{Cl}$ III $] \lambda 5517+37$} \\
$\mathrm{Ar}^{++}$ & {$[\mathrm{Ar}$ III $] \lambda \lambda 7136,7751$} \\
$\mathrm{Ar}^{+3}$ & {$[\mathrm{Ar}$ IV $] \lambda \lambda 4711+40$} \\
$\mathrm{Ar}^{+4}$ & {$[\mathrm{Ar} \mathrm{V}] \lambda 7005$} \\
\hline
\end{tabular}

Notes. ${ }^{(a)}$ Only for VLT H II region data.

Hernández-Martínez et al. 2009). Physical conditions and ionic abundances were computed using PYNEB, a new python-based code for the analysis of nebular data (Luridiana et al. 2015). The atomic data set used is listed in Table 2.

In general, a two-temperature model was adopted to derive ionic abundances, where $T_{\mathrm{e}}([\mathrm{O} \mathrm{III}])$ was used for high ionization species $\left(\mathrm{O}^{++}, \mathrm{Ne}^{++}, \mathrm{Ar}^{++}, \mathrm{Ar}^{+3}, \mathrm{He}^{+}\right.$, and $\left.\mathrm{He}^{++}\right)$, while $T_{\mathrm{e}}[\mathrm{N} \mathrm{II}]$ was used for the low-ionization ones $\left(\mathrm{N}^{+}, \mathrm{O}^{+}, \mathrm{S}^{+}\right.$, and $\left.\mathrm{S}^{++}\right)$. For $\mathrm{H} \mathrm{V}$, the weighted average of $T_{\mathrm{e}}([\mathrm{N} \mathrm{II}])$ and $T_{\mathrm{e}}([\mathrm{O} \mathrm{II}])$ was used to compute abundances of low ionization species. When only one temperature was available, it was used for all the ions. The density derived from the [S II] density sensitive line ratio was used in all the cases when available. In the objects where other diagnostics were available, the densities obtained were consistent within the uncertainties with the density derived from [S II] lines. When no density diagnostic was observed, a density of 1000 or $2000 \mathrm{~cm}^{-3}$ was adopted, depending on the case (see Tables A.2 and 4).

Uncertainties in the ionic abundances were calculated by applying Monte Carlo simulations. We generated 500 random values for each line intensity with a sigma equal to the uncertainty quoted for the line intensities. In Table 3 we show the list of lines used to derive ionic abundances.

Physical conditions and ionic abundances, with their respective errors, are presented in Table A. 2 for data from the VLT and the CFH telescope and for $\mathrm{HV}$ and $\mathrm{HX}$, and in Table 4 for data obtained with the GTC. Although we have estimated 
Table 4. Ionic abundances in NGC 6822 objects from GTC data.

\begin{tabular}{lcccccccc}
\hline \hline Object & PN2 & PN 8 & PN 11 & PN 13 & PN 23 & PN 24 & H II & H III \\
\hline$T_{\mathrm{e}}([\mathrm{O}$ III $])(\mathrm{K})$ & $18300 \pm 3800$ & $20300 \pm 3900$ & - & $20000 \pm 3000$ & $<14700$ & $16300 \pm 3300$ & $<16700$ & $12600 \pm 1000$ \\
$T_{\mathrm{e}}([\mathrm{N} \mathrm{II}])(\mathrm{K})$ & - & - & $17650 \pm 5750$ & - & - & $15850 \pm 8700$ & - & - \\
$n_{\mathrm{e}}([\mathrm{S} \mathrm{II}])\left(\mathrm{cm}^{-3}\right)$ & $750_{-600}^{+3000}$ & $6250:$ & $550_{-400}^{+1250}$ & $<100$ & $100_{-75}^{+300}$ & $200_{-140}^{+550}$ & $<100$ & $60_{-40}^{+100}$ \\
\hline Ion & \multicolumn{7}{c}{$12+\log \left(\mathrm{X}^{+i} / \mathrm{H}^{+}\right)$} \\
\hline $\mathrm{He}^{+}$ & $11.26 \pm 0.13$ & $10.57_{-0.11}^{+0.13}$ & - & $11.08 \pm 0.06$ & $10.89_{-0.16}^{+0.13}$ & $<11.15$ & $11.02 \pm 0.06$ & $10.96 \pm 0.02$ \\
$\mathrm{He}^{++}$ & $<9.73$ & $10.82 \pm 0.04$ & - & $<10.19$ & $>10.40$ & $10.79_{-0.08}^{+0.06}$ & - & - \\
$\mathrm{N}^{+}$ & $5.68_{-0.14}^{+0.22}$ & $5.84_{-0.15}^{+0.18}$ & $7.78_{-0.18}^{+0.21}$ & $5.41 \pm 0.20$ & $>6.62$ & $6.60:$ & $>6.18$ & $6.15_{-0.08}^{+0.10}$ \\
$\mathrm{O}^{+}$ & 6.72 & $5.83:$ & $<7.51$ & $6.71_{-0.18}^{+0.32}$ & $>7.43$ & - & $>7.49$ & $7.74_{-0.11}^{+0.14}$ \\
$\mathrm{O}^{++}$ & $7.47_{-0.15}^{+0.21}$ & $7.05_{-0.15}^{+0.26}$ & $7.35_{-0.24}^{+0.31}$ & $7.61_{-0.10}^{+0.19}$ & $>8.07$ & $7.83_{-0.17}^{+0.29}$ & $>7.07$ & $7.59_{-0.08}^{+0.12}$ \\
$\mathrm{Ne}^{++}$ & $6.74_{-0.30}^{+0.33}$ & $<6.08$ & - & - & - & - & $6.77_{-0.11}^{+0.15}$ \\
$\mathrm{~S}^{+}$ & $4.44_{-0.17}^{+0.25}$ & $4.45_{-0.35}^{+0.38}$ & $5.90 \pm 0.23$ & $4.78_{-0.11}^{+0.14}$ & $>5.51$ & $5.28_{-0.23}^{+0.31}$ & $>5.72$ & $5.85_{-0.07}^{+0.09}$ \\
$\mathrm{~S}^{++}$ & - & - & - & - & - & - & - & - \\
$\mathrm{Ar}^{++}$ & $4.83_{-0.16}^{+0.18}$ & $4.79_{-0.19}^{+0.22}$ & $5.77 \pm 0.28$ & $<5.22$ & $>5.56$ & $5.51_{-0.14}^{+0.20}$ & $>5.43$ & $5.65_{-0.07}^{+0.10}$ \\
$\mathrm{Ar}^{+4}$ & - & $4.98_{-0.26}^{+0.23}$ & - & - & - & - & - & - \\
\hline
\end{tabular}

Notes. Abundances in $12+\log \left(\mathrm{X}^{+i} / \mathrm{H}^{+}\right)$. Colons indicate errors larger than a factor of two.

Table 5. Total abundances in NGC 6822 from GTC data.

\begin{tabular}{lcccccccc}
\hline \hline Elem/object & PN2 & PN 8 & PN11 & PN13 & PN23 & PN24 & H II & H III \\
\hline $\mathrm{He}$ & $11.27 \pm 0.12$ & $11.01 \pm 0.05$ & - & $11.13 \pm 0.06$ & $11.01_{-0.13}^{+0.09}$ & $<11.30$ & $>11.02$ & - \\
$\mathrm{N}$ & $6.58_{-0.17}^{+0.17}$ & $7.28 \pm 0.32$ & $8.27_{-0.14}^{+0.27}$ & $6.39_{-0.23}^{+0.16}$ & $>7.38$ & - & $6.30:$ & $6.38 \pm 0.08$ \\
$\mathrm{O}$ & $7.55_{-0.18}^{+0.25}$ & $7.39_{-0.14}^{+0.25}$ & $7.74:$ & $7.69_{-0.10}^{+0.20}$ & $>8.23$ & $>7.83$ & $>7.63$ & $7.97_{-0.10}^{+0.13}$ \\
$\mathrm{Ne}$ & $6.76:$ & $<6.42$ & - & - & - & - & - & $6.88_{-0.12}^{+0.15}$ \\
$\mathrm{~S}$ & $5.56_{-0.22}^{+0.28}$ & $5.99:$ & - & $6.01 \pm 0.10$ & $>6.49$ & $>5.28$ & - & - \\
$\mathrm{Ar}$ & $4.97_{-0.13}^{+0.17}$ & $5.30_{-0.15}^{+0.20}$ & $6.50:$ & $<5.40$ & $>5.74$ & $>5.51$ & $5.48:$ & $5.69_{-0.07}^{+0.10}$ \\
\hline$L(\mathrm{H} \beta) / L_{\odot}$ & 13.95 & 21.79 & 0.22 & 56.85 & 3.27 & 7.07 & - & \\
\hline
\end{tabular}

Notes. Abundances in $12+\log (\mathrm{X} / \mathrm{H})$. Colons indicate errors larger than a factor of two.

some ionic abundances for the $\mathrm{H}$ II region $\mathrm{HII}$, these values are extremely uncertain because no electron temperature could be computed for this object, therefore it will not be considered for the discussion of total abundances in H II regions.

For elements heavier than He, we only considered abundances obtained from CELs. It is well known that abundances obtained from RLs are generally higher than those obtained from CELs (see Sect. 1), but although some oxygen RLs have been detected in H V and HX spectra (Peimbert et al. 2005), we do not detect oxygen RLs in the rest of our sample objects. Moreover, some authors claim that the ADF can be considered almost constant for $\mathrm{HII}$ regions in Galactic and extragalactic domain (García-Rojas \& Esteban 2007; Peña-Guerrero et al. 2012), but this is not necessarily true for PNe (McNabb et al. 2013), making any conclusion obtained from assuming a "canonical" ADF unreliable. However, if we consider that PNe in NGC 6822 follow the behaviour of Galactic and extragalactic $\mathrm{H}$ II regions and the bulk of PNe in our Galaxy, the effect of considering the ADF would be much lower than the quoted uncertainties in heavy element abundance ratios and would affect elemental abundances by adding $\sim 0.2$ dex to the CELs abundances.

\section{Total abundances and AGB nucleosynthesis}

To derive the total abundance of an element, we must add the determined ionic abundances and correct for the unseeing ions by employing ionization correction factors (ICF). Very recently, a new set of ICFs for PNe has been presented by Delgado-Inglada et al. (2014). These ICFs were computed from a large grid of photoionization models, covering a wide range of values in the parameter space, thus improving significantly the previous ICF sets for PNe in the literature. We checked the validity range of the ICFs, depending on the excitation of each PN. In the majority of the cases, the ICFs can be applied; however, four PNe (PN 12, PN 16, PN 18, and PN 21) showed $\mathrm{O}^{2+} /\left(\mathrm{O}^{+}+\mathrm{O}^{2+}\right) \geq$ 0.95 , which is out of the validity range of the ICF proposed by Delgado-Inglada et al. (2014) for N, S, and Ar. In such cases we compared the results obtained with the ICF provided by Delgado-Inglada et al. (2014) for $\mathrm{N}$ with the classical ICF scheme, i.e., $\mathrm{N} / \mathrm{O} \sim \mathrm{N}^{+} / \mathrm{O}^{+}$, and we found average differences of about $\pm 0.10 \mathrm{dex}$, which are within the uncertainties of the total abundance determinations; moreover, we do not find any unexpected behaviour of the abundances of Ar and S on these objects (see Sect. 5.3), and therefore, to have a homogeneous analysis, we applied the Delgado-Inglada et al. (2014) scheme to these objects. For H II regions we used the ICF expressions for midmetallicity $(7.6<(12+\log (\mathrm{O} / \mathrm{H})<8.2)$ proposed by Izotov et al. (2006). The intrinsic uncertainties of the Delgado-Inglada et al. (2014) ionization correction factors are not propagated in our analysis and only error propagation of the ionic abundances was considered in our Monte Carlo simulations. 
Total abundances for $19 \mathrm{PNe}$ are listed in Table A.3 for data from the VLT and CFH observations and in Table 5 for data from the GTC. In addition, the chemical abundances of four H II regions - H II 15 and H III observed by us and H V and H X from the work by Peimbert et al. (2005) for which we recalculated the abundances - have been included for comparison. The behaviour of elemental abundances are shown in Figs. 3-7, and they are discussed in the following. In these plots we have not included uncertain determinations of abundances (those with errors quoted as :) or the upper and lower limits to the abundances.

In Tables A. 3 and 5 we also list the total luminosity $L(\mathrm{H} \beta)$ of $\mathrm{PNe}$ in solar units for all the objects for which $\mathrm{F}(\mathrm{H} \beta)$ and $\mathrm{c}(\mathrm{H} \beta)$ are published. A distance of $459.19 \mathrm{kpc}$ was assumed for NGC 6822 (Gieren et al. 2006). After determining physical conditions and chemical abundances, in Fig. 2 we present some diagrams comparing the properties of PNe and $\mathrm{H}$ II regions that clearly show the differences between these two type of photoionized nebulae. It is evident that spectroscopic analysis allows a reliable classification on a very solid basis.

\subsection{O abundances}

In the upper panel of Fig. 3 , we present $12+\log (\mathrm{O} / \mathrm{H})$ vs. $\log \left(L(\mathrm{H} \beta) / L_{\odot}\right)$. In Tables A.3 and 5 , it is observed that PNe in NGC 6822 show $L(\mathrm{H} \beta)$ smaller than $60 L_{\odot}$, while H II regions (even the compact ones) are expected to be much brighter; for instance, the compact $\mathrm{H}$ II 15 has $L(\mathrm{H} \beta)$ larger than $300 L_{\odot}$. $L(\mathrm{H} \beta)$ for the most extended H II regions are not presented because only a fraction of the nebula was observed. Regarding $\mathrm{O}$ abundances, $12+\log (\mathrm{O} / \mathrm{H})$ values in $\mathrm{PNe}$ show a wide range from 7.39 to 8.27 , with the two brightest PNe showing intermedium $\mathrm{O}$ abundances. We do not find a particular trend between $L(\mathrm{H} \beta)$ and the $\mathrm{O} / \mathrm{H}$ abundance, although it is worth noticing that the faintest objetcs show very low $\mathrm{O}$ abundances. The same is observed in the lower panel of Fig. 3, where we plot $L([\mathrm{O}$ III $]$ 25007) instead of $L(\mathrm{H} \beta)$ and where no particular trend is again found.

In their Fig. 4, Corradi et al. (2015b) found for M 31 PNe that there seems to be a tendency toward decreasing metallicity with decreasing [O III] luminosity. They also seem to find a similar tendency when plotting M33 data from Magrini et al. (2009). Similar results have already been indicated by Stasińska et al. (1998) for the LMC, SMC, and the Milky Way. From our data, this tendency is not evident, although as pointed out above, the faintest $\mathrm{PNe}$ of our sample show low metallicities, while the bright ones have a wide spread in $\mathrm{O} / \mathrm{H}$ abundances.

\subsection{He, $N$, and $\mathrm{O}$}

Figure 4 shows the behaviour of $12+\log (\mathrm{N} / \mathrm{H})$ as a function of $12+\log (\mathrm{He} / \mathrm{H})$ (upper panel) and $\log (\mathrm{N} / \mathrm{O})$ ratio vs. $12+$ $\log (\mathrm{O} / \mathrm{H})$ (lower panel) for PNe (black dots) and H II regions (red stars). No correlation between these quantities is evident. In comparison with the $\mathrm{H}$ II regions, most of the PNe are both He-rich and N-rich, as occurs in all the samples analysed by many authors for different galaxies. This indicates that central stars of PNe are providing large amounts of $\mathrm{He}$ and $\mathrm{N}$ to the ISM as a consequence of the first and second dredge-up events experienced during the AGB phase. The second dredge-up occurs for the more massive stars with $M>3 M_{\odot}$. Such dredgeup processes enrich the stellar surface with $\mathrm{N}$ produced via the CNO cycle, but it is apparent from this figure that, since no correlation exists, the ON-cycle is not a significant contributor to
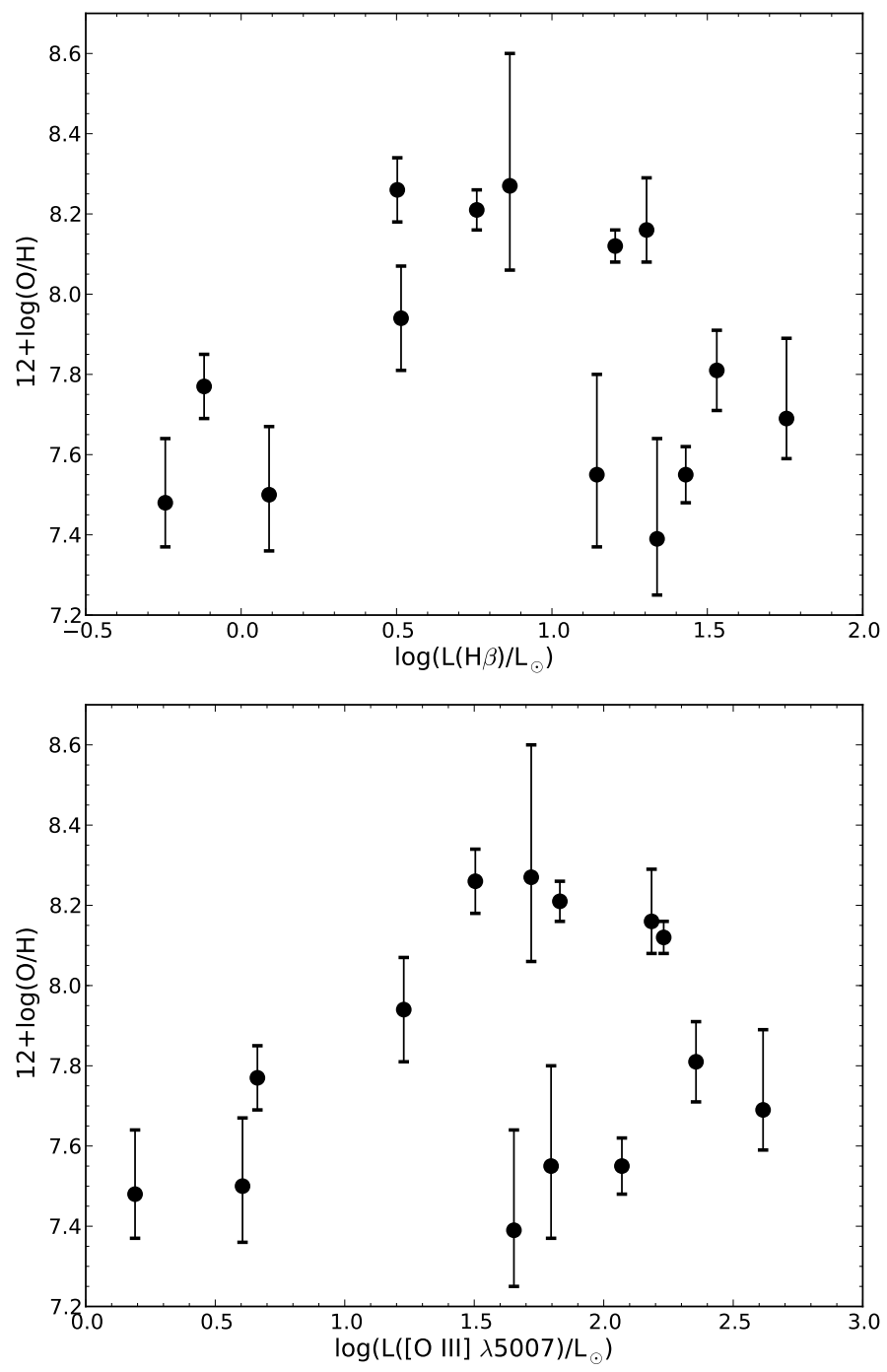

Fig. 3. Above: $12+\log (\mathrm{O} / \mathrm{H})$ vs. $L(\mathrm{H} \beta)$; below: $12+\log (\mathrm{O} / \mathrm{H})$ vs. $L([\mathrm{O}$ III $] 5007)$ for PNe in NGC 6822.

the $\mathrm{N}$ nucleosynthesis yield, and most of the $\mathrm{N}$ should be contributed by the $\mathrm{CN}$-cycle in these stars. However, a note of caution should be introduced here, because Karakas et al. (2009, and references therein) point out that $\mathrm{PNe}$ with rapid rotating progenitor stars can also reflect high $\mathrm{He}$ and $\mathrm{N}$ abundances in the gas owing to rotation changes the internal structure of the star, resulting in high $\mathrm{He} / \mathrm{H}$ and $\mathrm{N} / \mathrm{O}$ ratios in the surface, before the AGB phase. Extreme $\mathrm{N}$ enrichment is observed in some $\mathrm{PNe}$ (they would be Peimbert Type I PNe) whose central stars (in principle the most massive ones) produce extra $\mathrm{N}$ enrichment by experiencing envelope-burning conversion to $\mathrm{N}$ of dredgedup primary $\mathrm{C}$ (phenomenon known as "hot-bottom burning", hereinafter $\mathrm{HBB}$ ). Additionally, the role of the binary evolution of low-mass stars cannot be ruled out as the origin of a fraction of highly N-rich PNe (see e.g. Moe \& De Marco 2006, and references therein). If we consider Type I PNe as those with a N/O abundance ratio greater than 0.5 (see discussion in Hernández-Martínez et al. 2009, for adopting this value), we found that there are six Type I PNe in our sample, representing one third of the whole analysed sample, although two of them have very uncertain $\mathrm{N}$ and/or $\mathrm{O}$ abundances and are not represented in Fig. 4. Interestingly, in the lower panel of this figure, it is evident that these Type I PNe occur at any value of $\mathrm{O} / \mathrm{H}$ (that 

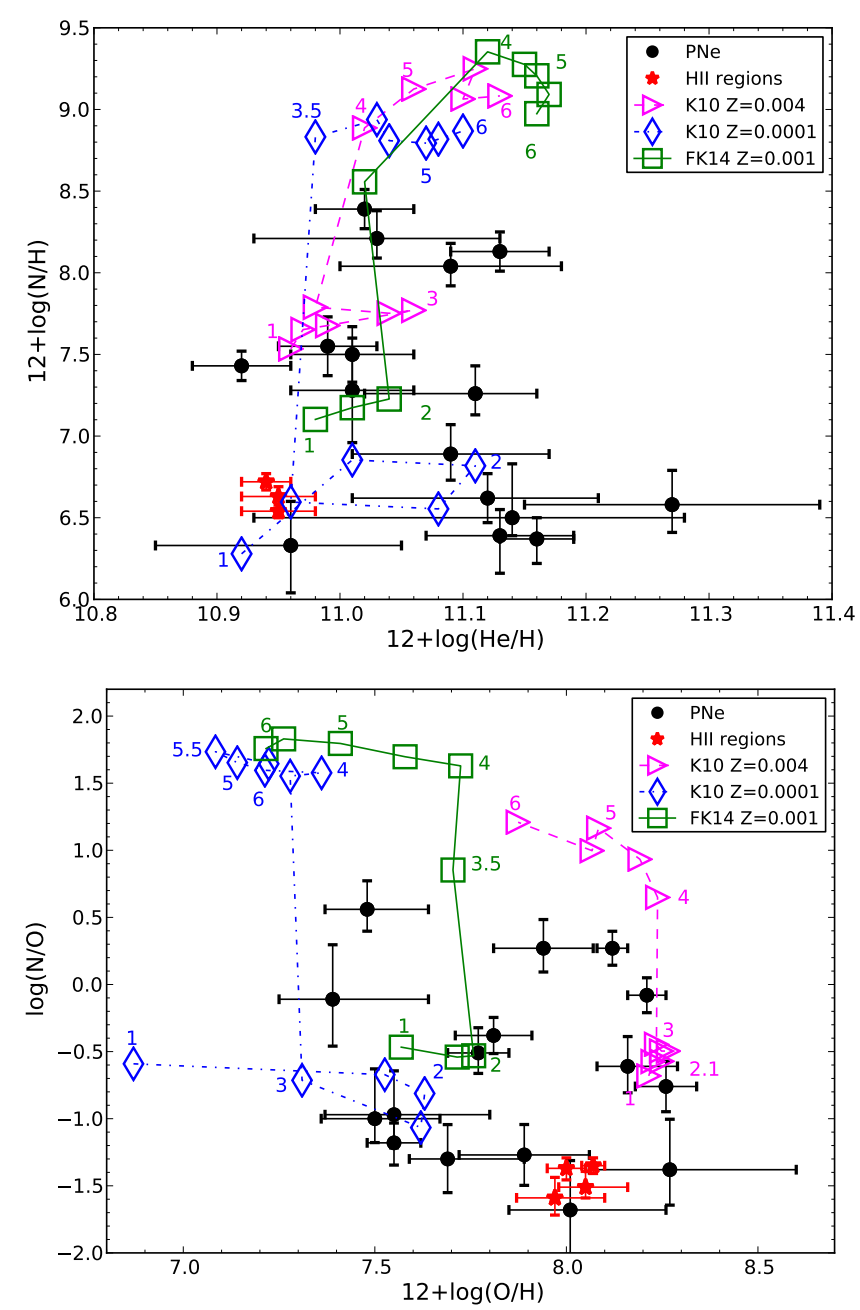

Fig. 4. $12+\log (\mathrm{N} / \mathrm{H})$ vs. $12+\log (\mathrm{He} / \mathrm{H})$ (upper panel) and $\log (\mathrm{N} / \mathrm{O})$ vs. $12+\log (\mathrm{O} / \mathrm{H})$ (lower panel) for PNe (in black) in NGC 6822. In red the $\mathrm{H}$ II regions. Stellar evolution models for different metallicities by Karakas (2010, K10) $(Z=0.0001$, blue diamonds; $Z=0.004$, magenta triangles) and Fishlock et al. (2014, FK14) $(Z=0.001$, green squares) have been included. Masses of the progenitors stars are shown for some models. See text for discussion.

is, at any metallicity), and they are not restricted to the young (supposedly more massive) high-metallicity objects.

As said above, to check the influence of the selected ICF scheme on our results, we also computed total $\mathrm{N}$ abundances using the classical ICF scheme of $\mathrm{N} / \mathrm{O} \sim \mathrm{N}^{+} / \mathrm{O}^{+}$, and we did not find any significant differences (on average, less than $\pm 0.1 \mathrm{dex}$ ) between using the Delgado-Inglada et al. (2014) scheme and the classical one, with the exception of $\mathrm{PN} 11$, whose N/O ratio decreases $\sim 0.26$ dex using the classical ICF scheme, but this object is not included in Fig. 4 because it shows a very uncertain $\mathrm{O} / \mathrm{H}$ ratio.

In Fig. 4 we have also included the behaviour predicted by the stellar evolution models by Karakas (2010) and Fishlock et al. (2014) for different metallicities. Model values correspond to the surface stellar abundances at the end of the AGB phase. The initial masses of stars have been tagged. In the upper panel of this figure, it is apparent that our objects have larger $\mathrm{He} / \mathrm{H}$ abundances than what is predicted by the models. Only models with metallicity $Z=0.0001$ would be in agreement with the He abundance shown by PNe with $12+\log (\mathrm{N} / \mathrm{H})$ lower than 7.0.

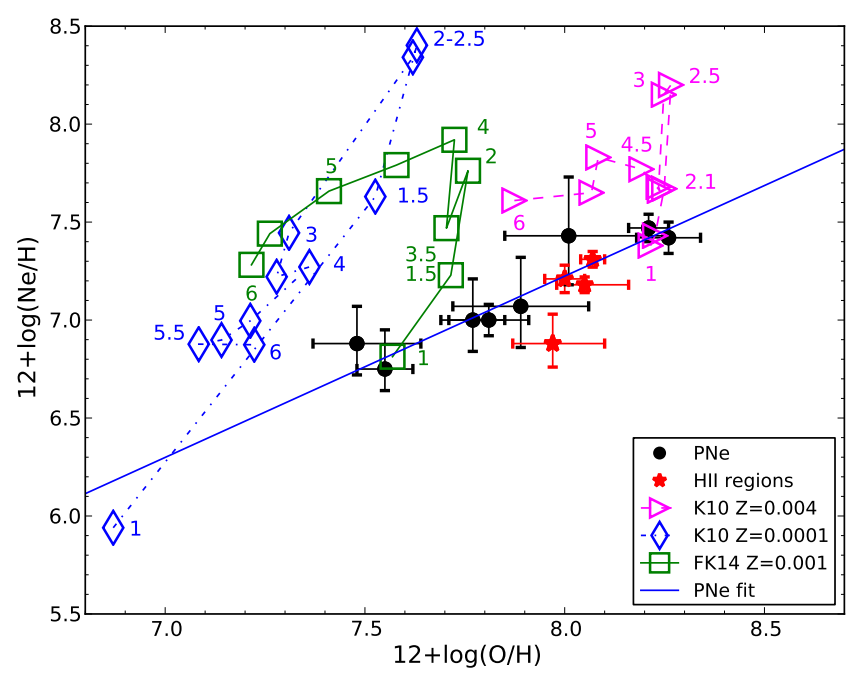

Fig. 5. $12+\log (\mathrm{Ne} / \mathrm{H})$ vs. $12+\log (\mathrm{O} / \mathrm{H})$, for $\mathrm{PNe}$ (in black) in NGC 6822. In red the HII regions. The linear fit (including only PNe data) corresponds to $12+\log (\mathrm{Ne} / \mathrm{H})=(0.925 \pm 0.071) \times(12+\log$ $(\mathrm{O} / \mathrm{H}))-(0.176 \pm 0.566)$, with $r=0.88$. Stellar evolution models for different metallicities, by Karakas $(2010, \mathrm{~K} 10)(Z=0.0001$, blue diamonds; $Z=0.004$, magenta triangles) and Fishlock et al. (2014, F14) $(Z=0.0001$, green squares) have been included. See text for discussion.

In the lower panel of Fig. 4, it is found that our objects appear well demarcated by models with $Z$ between 0.0001 and 0.004 . As expected, models predict that more massive stars produce larger amounts of $\mathrm{N}$, similar to the values shown by Type I PNe. According to these models, our objects would have had initial masses lower than $4 M_{\odot}$; however, it should be noticed that no model predicts the low $\mathrm{N} / \mathrm{O}$ ratio $(\log (\mathrm{N} / \mathrm{O})>-1.0)$ shown by several of our PNe. Such stars should have had initial masses lower than $1 M_{\odot}$, or the stellar evolution models could be predicting yields for $\mathrm{N}$ that are too large. The behaviour of these models is discussed further in Sect. 5.4.

\subsection{The alpha elements: $\mathrm{Ne}, \mathrm{Ar}$, and $\mathrm{S}$}

The $\mathrm{Ne} / \mathrm{H}$ vs. $\mathrm{O} / \mathrm{H}$ abundance behaviour is shown in Fig. 5. Values derived for PNe and $\mathrm{H}$ II regions are presented. A tight correlation is found between $\mathrm{Ne}$ and $\mathrm{O}$ abundances as occurs in any other sample of PNe and H II regions (Henry 1989; GarcíaHernández \& Górny 2014). This has been interpreted as Ne not being strongly altered by AGB evolution so that $\mathrm{O}$ and $\mathrm{Ne}$ grow in lock-step during the chemical evolution of galaxies. In the case of our PNe sample, the slope of the correlation is $0.93 \pm 0.07$, which is similar to the slopes found for other PN samples; for instance, Leisy \& Dennefeld (2006) report slopes of 1.13 for PNe in the LMC and 1.01 in the SMC.

Similar to what has been done with $\mathrm{N}$, we checked the $\mathrm{Ne} / \mathrm{H}$ ratio obtained using the ICF scheme by Kingsburgh \& Barlow (1994). The differences between this ICF scheme and the one assumed in this work (Delgado-Inglada et al. 2014) are, on average, much smaller than the derived uncertainties $(<0.05 \mathrm{dex})$ with the exception of two objects, PN 17 and PN 6, for which the Kingsburgh \& Barlow scheme gives $\mathrm{Ne} / \mathrm{H}$ ratios that are 0.48 and 0.26 dex larger, respectively. PN 6 is not shown in Fig. 5 owing to its uncertain $\mathrm{Ne} / \mathrm{H}$ ratio.

In Fig. 5 we have also included the predictions of stellar evolution models by Karakas (2010) and Fishlock et al. (2014) for different metallicities. These models consider the yields of all the Ne isotopes: ${ }^{20} \mathrm{Ne},{ }^{21} \mathrm{Ne}$, and ${ }^{22} \mathrm{Ne}$. It is worth recalling that ${ }^{20} \mathrm{Ne}$ is the most abundant isotope, and it is not significantly 


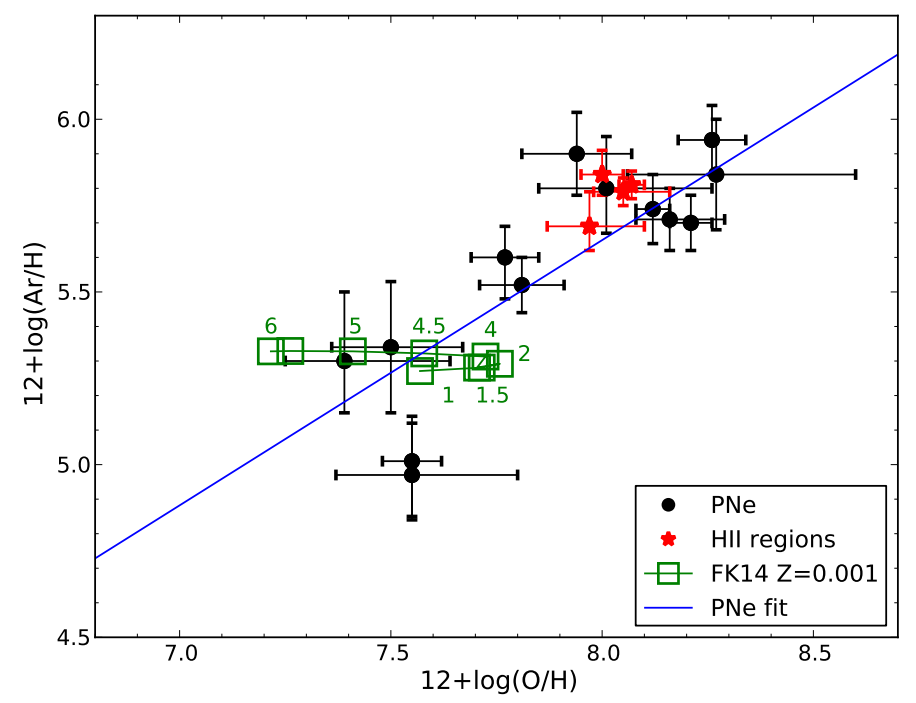

Fig. 6. Behaviour of $12+\log (\mathrm{Ar} / \mathrm{H})$ vs. $12+\log (\mathrm{O} / \mathrm{H})$. In red the $\mathrm{H}$ II regions. The linear fits, including only $\mathrm{PNe}$, corresponds to $12+$ $\log (\mathrm{Ar} / \mathrm{H})=(-0.494 \pm 0.512)+(0.768 \pm 0.064) \times(12+\log (\mathrm{O} / \mathrm{H}))$, with $r=0.79$. The stellar evolution models for $Z=0.001$ by Fishlock et al. $(2014$, F14) are included.

modified by nucleosynthesis during the AGB phase, but two $\alpha$ captures may transform a ${ }^{14} \mathrm{~N}$ into a ${ }^{22} \mathrm{Ne}$ that could be mixed to the surface in the third dredge-up episode, increasing the total $\mathrm{Ne}$ abundance. Interestingly, the models predict large $\mathrm{Ne}$ increases at very low metallicities. ${ }^{22} \mathrm{Ne}$ is efficiently produced and brought to the surface in stars with initial masses between $\sim 2$ and $4 M_{\odot}$, depending on the metallicity. Stars with higher masses are less efficient because ${ }^{22} \mathrm{Ne}$ diminishes as a consequence of an $\alpha$ capture that destroys it. Our objects are delimited by models with $Z$ between 0.001 and 0.004 . In this case we found that models predict excess of $\mathrm{Ne}$, unless our objects have had initial masses lower than 1.5 or $2 M_{\odot}$, which contradicts the results from the $\mathrm{N} / \mathrm{O}$ vs. O/H diagram using the same set of models (see Fig. 4 in previous section). In Sect. 5.4 this subject is discussed more deeply.

The behaviour of $\mathrm{Ar} / \mathrm{H}$ vs. $\mathrm{O} / \mathrm{H}$ is presented Fig. 6. A correlation is also found for these elemental abundances. Although it is not as tight as in the case of $\mathrm{Ne} / \mathrm{H}$ vs. $\mathrm{O} / \mathrm{H}$, it shows that $\mathrm{O}, \mathrm{Ne}$, and $\mathrm{Ar}$ are elements that evolve closely in lock-steps. Data for $\mathrm{H}$ II regions have been included in this diagram (red dots). In this case the slope of the correlation (computed including PNe only) is $0.768 \pm 0.064$, significantly far from 1 , which could indicate that $\mathrm{O}$ abundances are too large at the high-metallicity end, as would occur if $\mathrm{O}$ is enriched by stellar nucleosynthesis.

The predictions of evolution models by Fishlock et al. (2014) at $Z=0.001$ are included in the graph. These models show clearly that $\mathrm{Ar}$ is not modified by stellar nucleosynthesis, independently of the initial stellar mass. In this case, the $12+$ $\log (\mathrm{Ar} / \mathrm{H})$ abundance has an almost constant value around 5.3.

$\mathrm{S} / \mathrm{H}$ vs. $\mathrm{O} / \mathrm{H}$ abundances are presented in Fig. 7. In this graph no correlation of $\mathrm{S}$ with $\mathrm{O}$ is found, although it should be expected, because $\mathrm{S}$ is also an $\alpha$ element produced in similar processes to $\mathrm{O}, \mathrm{Ne}$, and Ar. Probably this has to do with the difficulty of deriving the $S$ total abundance for which no appropriate ICF exists (Henry et al. 2004). In this graph we see that $\mathrm{S} / \mathrm{H}$ values in PNe spread in a wide range, and it is also seen that $\mathrm{H}$ II regions show a $\mathrm{S} / \mathrm{H}$ ratio similar to many $\mathrm{PNe}$, but an important fraction of $\mathrm{PNe}$ present lower $\mathrm{S} / \mathrm{H}$ than the values in $\mathrm{HII}$ regions. Therefore the phenomenon known as "sulphur anomaly"

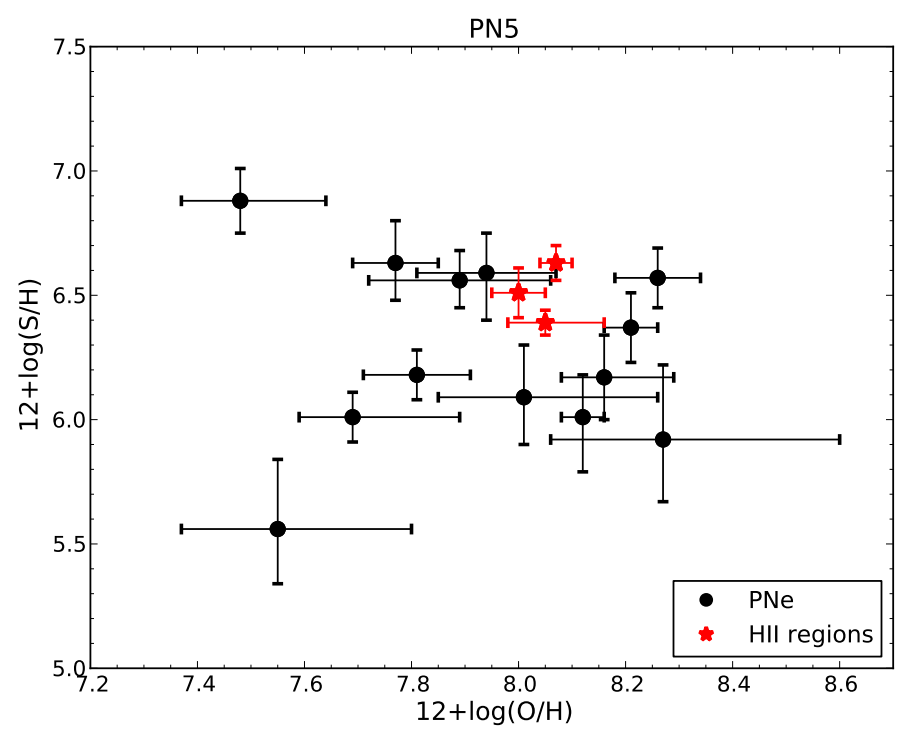

Fig. 7. Behaviour of $12+\log (\mathrm{S} / \mathrm{H})$ vs. $12+\log (\mathrm{O} / \mathrm{H})$, for $\mathrm{PNe}$ in NGC 6822. In red the HII regions. No correlation exists. The H II regions present larger $\mathrm{S}$ abundances than many $\mathrm{PNe}$.

(consisting in PNe showing sustantially lower S abundance than $\mathrm{H}$ II regions at a given metallicity) is present in our PN sample. The reasons behind this phenomenon are still not clear, although as said above, it has been attributed mainly to deficient ICFs for calculating $\mathrm{S}$ abundance, particularly, to the uncertain contribution of $\mathrm{S}^{3+}$ to the total $\mathrm{S}$. In our case, the use of the new ICFs proposed by Delgado-Inglada et al. (2014) has not improved the situation. On the other hand, some authors claim that the sulphur anomaly can be explained by the depletion of $\mathrm{S}$ into dust, especially in C-rich dust PNe (García-Hernández \& Górny 2014). Computing abundances from $\mathrm{S}$ lines in IR spectra, where $S^{3+}$ lines can be measured, would shed some light on this problem.

\subsection{Testing other stellar evolution models}

As said above, the comparison of the observed N/O abundances with predictions of stellar evolution models by Karakas (2010) and Fishlock et al. (2014) indicates that the initial masses of the observed PNe were lower than $4 M_{\odot}$, contradicting the predictions of the same models for $\mathrm{Ne} / \mathrm{H}$ vs. O/H (Fig. 5), from which the initial masses should have been lower than $2 M_{\odot}$. Therefore we have decided to analyse the behaviour of models more deeply.

Karakas \& Lattanzio (2003) have compared the Ne yield of their models with abundances of PNe of the Milky Way $(Z=$ 0.02) and the Large Magellanic Cloud $(Z=0.008)$ to find that models agree with the observations except for the case of models with initial mass of $3 M_{\odot}$ and $Z=0.008$, which seem to predict too much $\mathrm{Ne}$.

From the analysis of a sample of Galactic disk and bulge double chemistry (DC), O-rich dust (OC), and C-rich dust (CC) PNe, García-Hernández \& Górny (2014) found that the average $\mathrm{Ne} / \mathrm{O}$ at $Z=0.02$ for their DC and OC PNe was slightly larger than those predicted by the models by Karakas (2010) without a partial mixed zone (PMZ); therefore, they conclude that including and varying the size of a PMZ can solve the observed behaviour. However, that is exactly the opposite of what we found in our sample. For our objects (which have $Z<0.004$ ), we found that models with initial mass higher than $2 M_{\odot}$ show too much $\mathrm{Ne}$, in comparison with observations. We consider that 

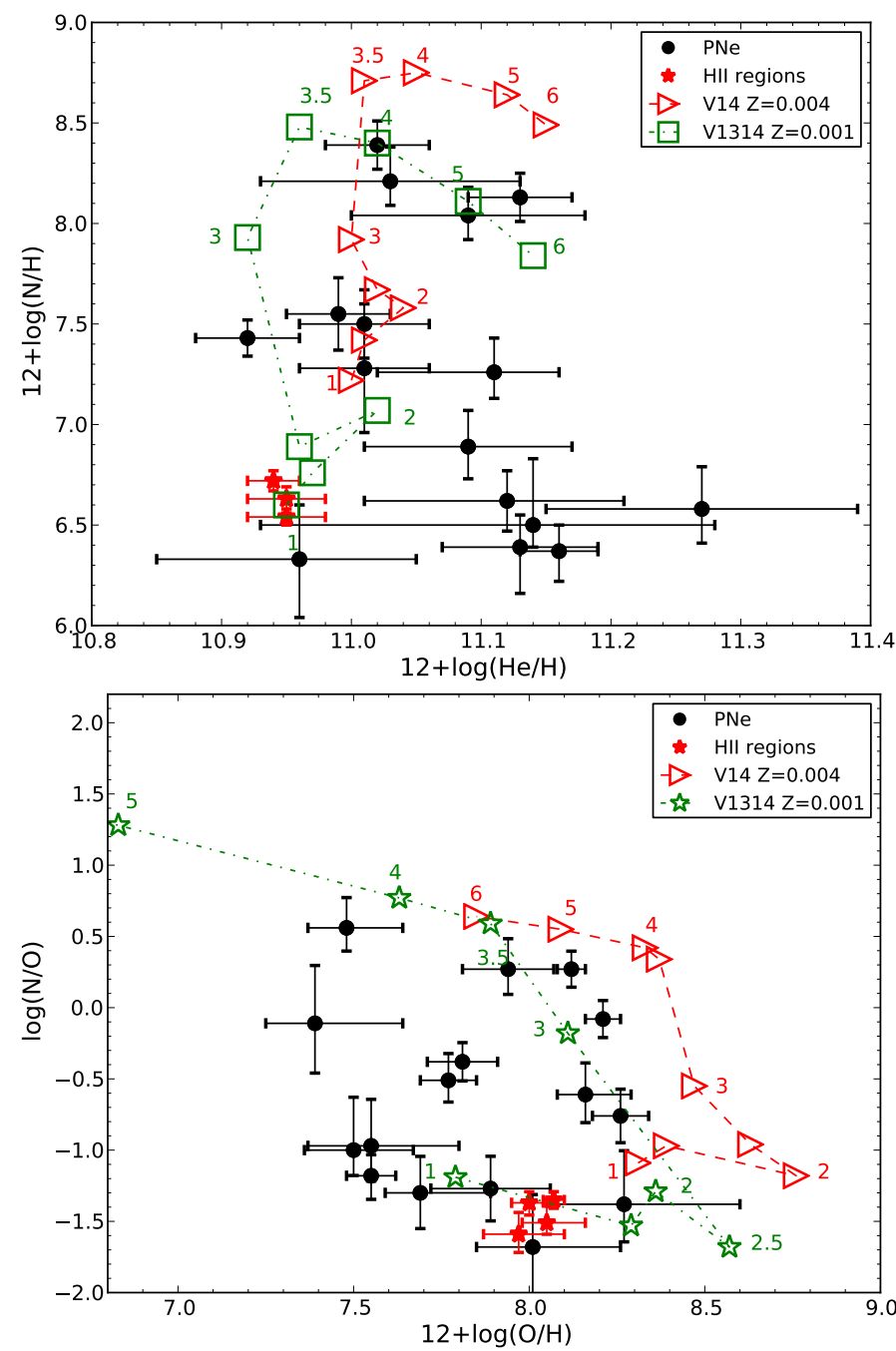

Fig. 8. Same as Fig. 4 but showing the predictions of stellar evolution models for $Z=0.001$ (green squares) by Ventura et al. (2013, 2014b, V1314) and $Z=0.004$ (red triangles) by Ventura et al. (2014a, V14). See text for discussion.

it is possible that such models are producing an excess of ${ }^{22} \mathrm{Ne}$ or that the efficiency of the third dredge-up should be diminished in those stars.

Very recently, Ventura et al. (2015) and Dell'Agli et al. (2015) have used a new generation of AGB stellar models that include dust formation in the stellar winds to constrain $\mathrm{CNO}$ abundances of PNe in the Magellanic Clouds, which have a metallicity slightly higher than but comparable to that of NGC 6822. These models were developed using the ATON evolutionary code, which stands apart from others in that it uses the Blöcker (1995) mass loss prescription and the full spectrum of turbulence (FST) convective model (Canuto \& Mazzitelli 1991). These assumptions concern the overshoot of the convective core during the core H-burning phase and lead to a less efficient dredge-up and to a lower threshold mass for the activation of the HBB. A detailed description of these models are given in Ventura et al. (2014a) for $Z=0.004$, in Ventura et al. (2013) for $Z=0.001$ and $M>3 M_{\odot}$, and in Ventura et al. (2014b) for $Z=0.001$ and $M \leq 3 M_{\odot}$.

In Figs. 8 and 9 we explore the behaviour of $12+\log (\mathrm{N} / \mathrm{H})$ vs. $12+\log (\mathrm{He} / \mathrm{H})$ and $\log (\mathrm{N} / \mathrm{O})$, and $12+\log (\mathrm{Ne} / \mathrm{H})$ vs. 12 $+\log (\mathrm{O} / \mathrm{H})$ with models by Ventura et al. $(2013,2014 \mathrm{~b})$ for $Z=0.001$ and Ventura et al. (2014a) for $Z=0.004$. It is found

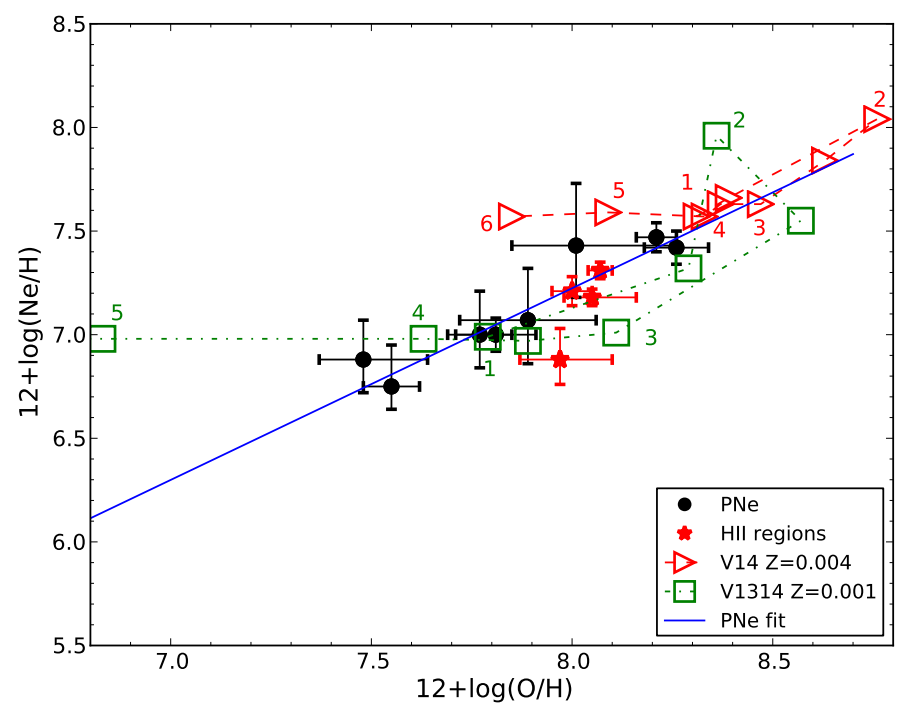

Fig. 9. Same as Fig. 5 but showing the predictions of stellar evolution models for $Z=0.001$ (green squares) by Ventura et al. (2013, 2014b, V1314) and $Z=0.004$ (red triangles) by Ventura et al. (2014a, V14). See text for discussion.

that they predict lower N/O ratios for intermediate-mass progenitors stars than the models by Karakas (2010) and Fishlock et al. (2014). Additionally, the lowest N/O ratios are reproduced by stars with initial masses lower than $2.5 M_{\odot}$. It is important to stress than in the $Z=0.001$ models, the HBB destroys $\mathrm{O}$ very efficiently in the more massive progenitors. In our sample there are several objects at $12+\log (\mathrm{O} / \mathrm{H})<7.8$ that are not reproduced by the set of models shown here. Probably these objects could be reproduced by models at $Z=0.0001$. In Fig. 8 (above), it is observed that Ventura et al. models do not reproduce the large $\mathrm{He} / \mathrm{H}$ abundance found in the $\mathrm{PNe}$ with $12+\log (\mathrm{N} / \mathrm{H})<$ 7.0).

Similar to what it was found for N, in Fig. 9 the observed $\mathrm{Ne}$ abundances are consistent with models of stellar progenitors with masses up to $4-5 M_{\odot}$, which is in overall agreement with what we found in Fig. 8. This is because dredge-up events in Ventura et al. (2015) models are less efficient than the Karakas ones.

On the other hand, we checked the evolution of the surface abundances obtained from the Fishlock et al. (2014) models during the different thermal pulses (TP) and dredge-up events. In Fig. 10 we show the behaviour of the $\mathrm{N} / \mathrm{O}$ and $\mathrm{Ne} / \mathrm{H}$ surface abundances for models with initial masses of $3.5,4$, and $5 M_{\odot}$ at different steps, from the first TP to the final surface abundances in steps of $10 \mathrm{TP}$, and we found that if the models are stopped significantly before (the amount of mass loss changes significantly) they can also reproduce the observed behaviour in N/O.

A deeper discussion of this subject is beyond the scope of this paper, but it would be important to analyse the predictions of models in comparison with the chemical abundances of different elements ( $\mathrm{C}, \mathrm{N}, \mathrm{O}, \mathrm{Ne}, \mathrm{Ar}$, etc.) observed in $\mathrm{PNe}$ at different metallicities, looking for a good agreement between observations and theory.

\subsection{PNe populations}

From a sample of 11 objects Hernández-Martínez et al. (2009) reported the presence of two PN populations, one older, of low metallicity with $12+\log (\mathrm{O} / \mathrm{H})$ values lower than 7.9 , and one younger, of high metallicity, including $\mathrm{PNe}$ showing $12+\log$ 

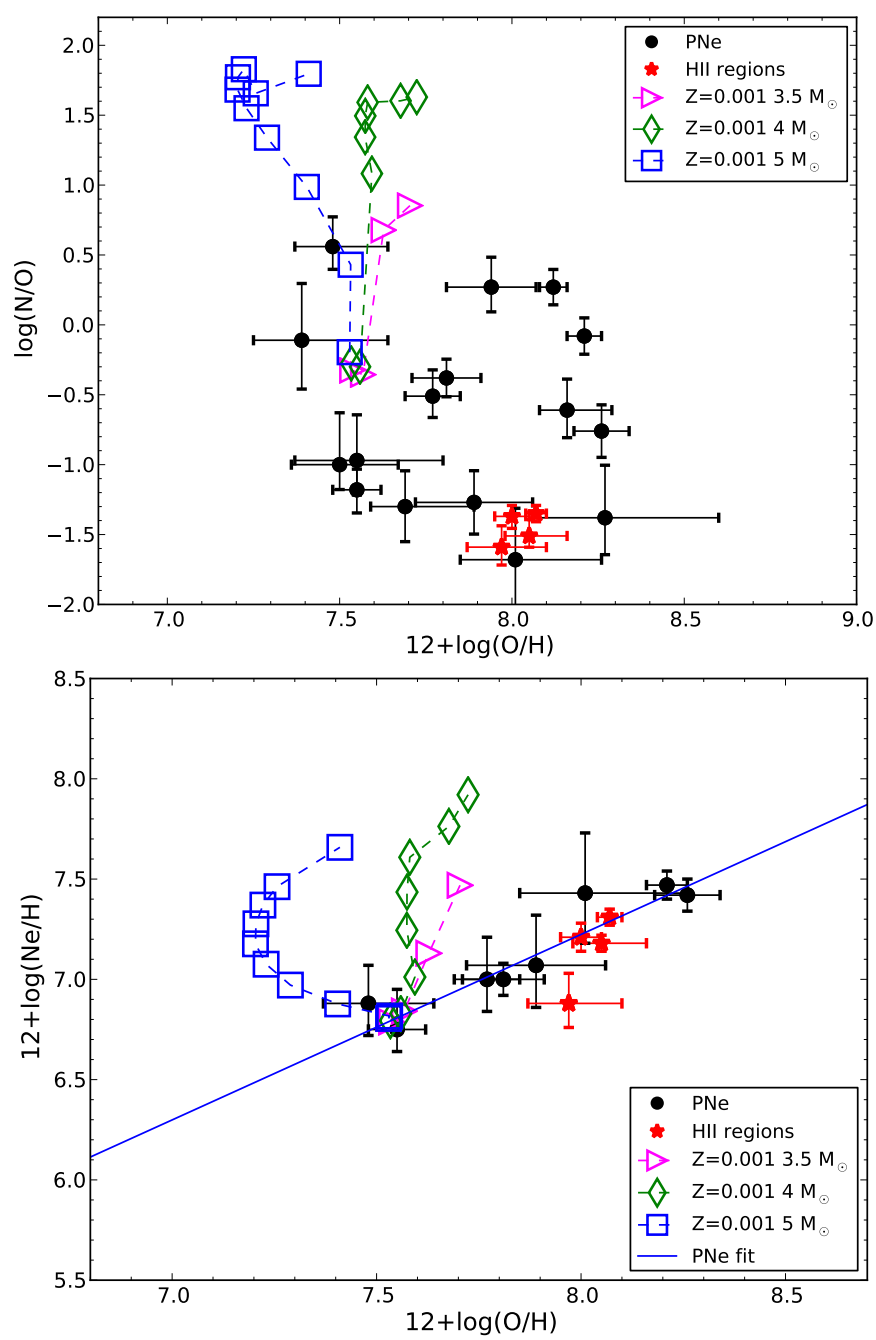

Fig. 10. Evolution of the surface abundances for models with $Z=0.001$ and initial masses of 3.5, 4, and $5 M_{\odot}$ of Fishlock et al. (2014). First point shows abundances after first TP and the last point represents the final surface abundances of the AGB model. The step between points is $\sim 10 \mathrm{TP}$.

$(\mathrm{O} / \mathrm{H})$ abundances larger than 8.0 and similar to the $\mathrm{H}$ II region abundances (the four H II regions presented in Tables 7 and 8 show a very similar $12+\log (\mathrm{O} / \mathrm{H})$ value with an average of $8.02 \pm 0.05)$. In our extended PN sample, such a segregation does not seem so evident. However, there is only one PN with $12+$ $\log (\mathrm{O} / \mathrm{H})$ in the range from 7.9 to 8.0 , therefore our sample still supports the idea of having two PN populations on the basis of $\mathrm{O} / \mathrm{H}$ abundances.

By analysing the behaviour of Ar abundances in Fig. 6, two PN populations can also be differentiated, one showing $12+$ $\log (\mathrm{Ar} / \mathrm{H})$ lower than 5.7 and the other $\mathrm{Ar} / \mathrm{H}$ similar to the values in H II regions, which on average is $5.80 \pm 0.10$.

As said in the Introduction, Carigi et al. (2006) used Wyder (2003) data and proposed a star formation history representative of the whole galaxy. In their Fig. 4 presenting the star formation rate (SFR), a clear burst appears between 6 and $8 \mathrm{Gyr}$, followed by a fall in the SFR lasting up to about $10 \mathrm{Gyr}$, when a second star formation episode starts. These two episodes of star formation coincide with the ages assigned by Hernández-Martínez et. al (2011) to the PN two populations, between 3 and 9 Gyr for the older one and ages under $3 \mathrm{Gyr}$ for the younger one. These PN ages were obtained from Allen

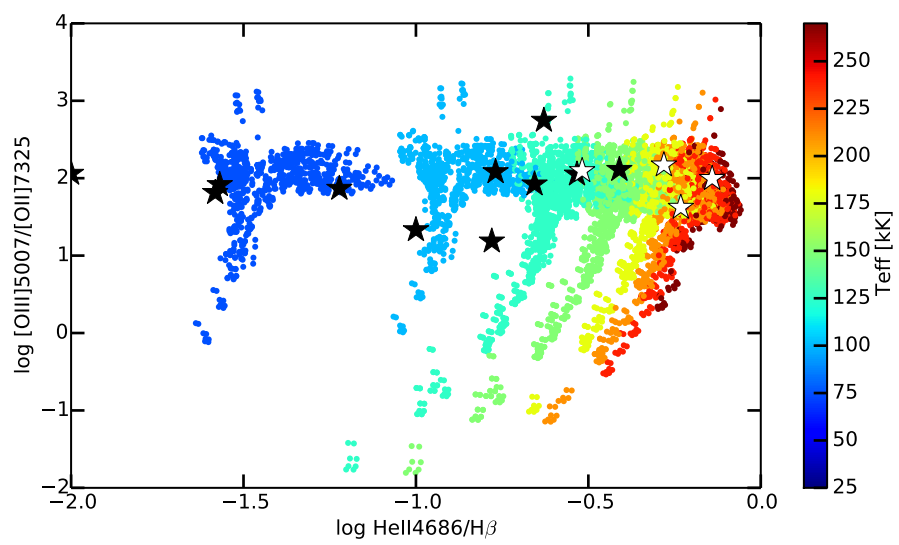

Fig. 11. $\lambda 5007 /[\mathrm{O}$ II $] \lambda 7325$ vs. He II $\lambda 4686 / \mathrm{H} \beta$ as obtained from the Mexican Million Models data base by Morisset et al. (2015). Stellar temperatures can be obtained from this plot. Many of our PNe have stars with $T$ higher than $10^{5} \mathrm{~K}$. The Type I PNe are indicated in white.

et al. (1998, and references therein). Additionally, according to Rodríguez-González et al. (2015), the presence of a dip in the PNLF in NGC 6822 indicates that two important episodes of star formation should have occurred in order to obtain such a dip in the PNLF. Therefore, our finding of two PN populations in NGC 6822 and their ages corroborate the two star formation episodes found in the SFR by Carigi et al. (2006).

Regarding the Ar abundances, as expected within uncertainties, no PN shows Ar/H larger than $\mathrm{H}$ II regions. However this is not the case of $\mathrm{O}$, for which a few $\mathrm{PNe}$ have $\mathrm{O} / \mathrm{H}$ slightly larger than H II regions (see Fig. 6). Similar to the slope of the $\mathrm{Ar} / \mathrm{H}$ vs. $\mathrm{O} / \mathrm{H}$ correlation, this could indicate $\mathrm{O}$-enrichment in the PN shell due to nucleosynthesis in the stellar interior. Such an enrichment has been predicted by some stellar evolution models for low-metallicity stars (see e.g. Marigo 2001) and from some nucleosynthesis models including extra mixing mechanism (Pignatari et al. 2013). Peña et al. (2007) find O-enrichment in the PNe of the low-metallicity irregular galaxy NGC 3109. Such an O-enrichment has also been found in some PNe of the Milky Way showing C-rich dust (Delgado-Inglada et al. 2015), and it is attributed to an efficient third dredge-up episode. In case of $\mathrm{O}$-enrichment, $\mathrm{O}$ abundance does not trace the metallicities of objects and, therefore Ar or other elements, such as $\mathrm{Cl}$, are better indicators of initial metallicities.

\section{Results and discussion}

From observations obtained with the GTC, VLT, and CFH telescopes, we have analysed the spectrophotometric data of $22 \mathrm{PNe}$ in NGC 6822. This represents $84 \%$ of the total sample of $26 \mathrm{PN}$ candidates known in this galaxy. We have confirmed the PN nature of $73 \%$ of the sample. Physical conditions and chemical abundances (in particular helium, nitrogen, oxygen, argon, and sulphur) were derived for $19 \mathrm{PNe}$. In addition, the chemical abundances of four H II regions have been included for comparison: H II 15 and H III observed by us and H V and H X from data by Peimbert et al. (2005). In the following we discuss the main results of our analysis.

A large number of Peimbert Type I PNe were found. (Following Hernández-Martínez et al. 2009, Peimbert Type I PNe were selected as those having N/O abundance ratio larger than 0.5.) These are PN 5, PN 8, PN 11, PN 14, PN 16, and 


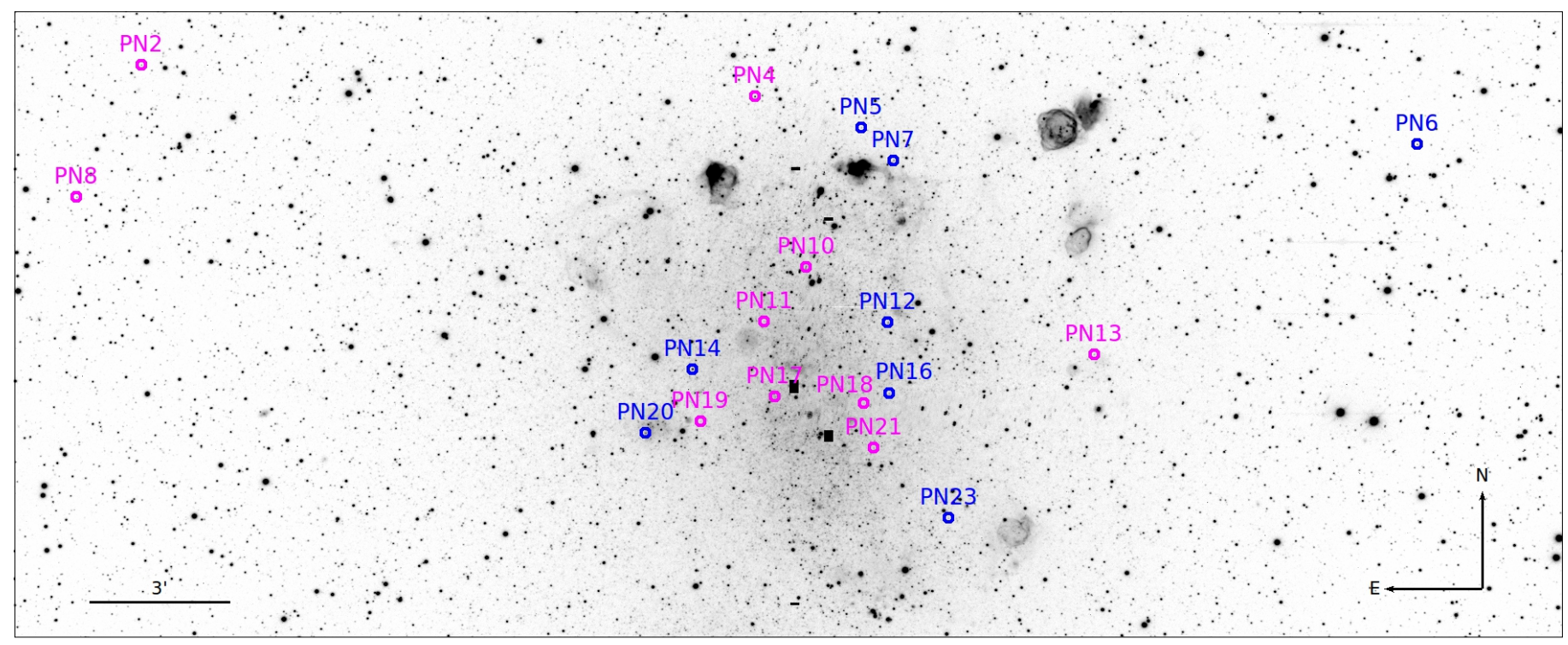

Fig. 12. Distribution of PNe in NGC 6822 in an $\mathrm{H} \alpha$ image. In pink the objects with low metallicity $(12+\log (\mathrm{O} / \mathrm{H})<8$ and $12+\log (\mathrm{Ar} / \mathrm{H})<$ 5.7); in blue, the high-metallicity ones $(12+\log (\mathrm{O} / \mathrm{H})>8$ and $12+\log (\mathrm{Ar} / \mathrm{H})>5.7$. PN 6, with $12+\log (\mathrm{Ar} / \mathrm{H})$ of 5.71 is marginally a young object).

PN 18, representing over $31 \%$ of the analysed sample (very probably PN 25 is also a Type I PN; but it is not included here since we cannot calculate its chemical abundances, and it is potentially affected by atmospheric diffraction effects, see Sect. 2). This large number resembles the number of Type I PNe found in the galactic bulge, M31 and the LMC (Stasińska et al. 1998). We recall that Type I PNe are He- and N-rich objects and they originated in stars that are more massive than in other PN types. Therefore it seems that we are detecting a large number of PNe produced by the more massive central stars, and the same is true for other galaxies. From the comparison of our N/O values and the predictions of stellar evolution models (Figs. 4 and 8), it is found that the initial masses of the central stars were lower than $4 M_{\odot}$ and that Type I PNe had masses between 3 and $4 M_{\odot}$.

Highly ionized $\mathrm{PNe}$ with a ionic fraction $\mathrm{He}^{++} / \mathrm{He} \geq 0.10$ are PN 4, PN 5, PN 8, PN 12, PN 14, PN 16, PN 23, PN 24, PN 13, PN 18, and PN 19, representing almost $60 \%$ of the sample. This high percentage of $\mathrm{He}^{++} / \mathrm{He}$ implies the presence of a very hot central star. Models from the ample grid computed by Morisset et al. (2015) (Mexican Million Models data base, 3MdB) have been used to analyse this. We chose the 'Realistic' and matterbounded PNe models (PNE-2014, Delgado-Inglada et al. 2014) that use a black body (BB) for the spectral energy distribution of the ionizing star. From this grid we have constructed Fig. 11, showing the behaviour of our objects in comparison with photoionization models, in an [O III $] \lambda 5007 /[\mathrm{O}$ II $] \lambda 7325$ vs. He II $\lambda 4686 / \mathrm{H} \beta$ diagram. The effective temperature for the models is shown in the colour bar. Our sample of PNe with $\mathrm{He}^{++} / \mathrm{He}$ $\geq 0.10$ corresponds to objects with effective temperatures over $100000 \mathrm{~K}$. It is important to notice that we have a limited number of objects with [O II] $\lambda 7325$, but this is not a problem because in this figure we can clearly see that the ratio He II $\lambda 4684 / \mathrm{H}_{\beta}$ is the most important parameter for determining the effective temperature, which is almost independent of the $[\mathrm{O}$ III $] \lambda 5007 /[\mathrm{O}$ II $] \lambda 7325$ ratio. The large number of highly ionized PNe in NGC 6822 seems unusual when compared to the PNe in our galaxy, but it is similar to what is found in the LMC (Stasińska et al. 1998). Type I PNe have been marked in green in this figure. It is evident that these $\mathrm{PNe}$ present

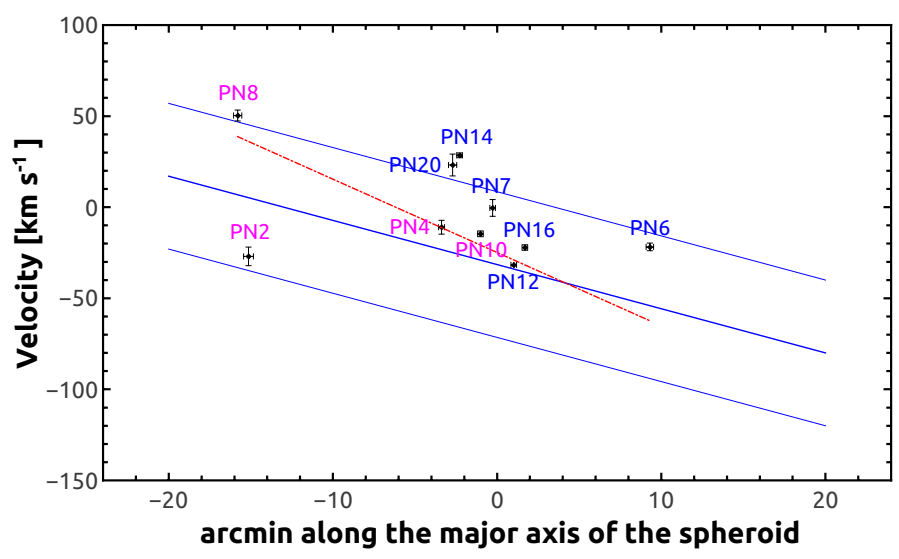

Fig. 13. Position-velocity of PNe in NGC 6822 with abundances determined, adapted from Flores-Durán et al. (2014). The position is projected on the long axis of the stellar spheroid. In pink the old objects $(12+\log (\mathrm{Ar} / \mathrm{H}) \leq 5.7)$; in blue the young objetcs.

the highest He II $\lambda 4686 / \mathrm{H} \beta$ line intensity ratios and the highest stellar temperatures, which are in the range from 140 to $230 \mathrm{kK}$. If we compare such temperatures with the evolutionary tracks presented by Vassiliadis \& Wood (1994) for the SMC metallicity (their Fig. 5), we conclude that the three hottest stars had initial masses higher than $2.5 M_{\odot}$ (and would have present core masses higher than $0.69 M_{\odot}$ ). This agrees with the initial masses derived for Type Is from the N/O ratio (Figs. 4 and 8).

Therefore our PN sample seems to have some biases: we find too many Type I PNe and very hot central stars (as compared to the galactic values). Our PN candidates were selected from a sample of [O III $] \lambda 5007$ - and $\mathrm{H} \alpha$ - emitting objects (Hernández-Martínez \& Peña 2009). Actually to be certain they are PN and to eliminate compact H II regions, Ciardullo et al. (2002) suggested selecting PN candidates because they have [O III] $\lambda 5007 / \mathrm{H} \alpha$ ratios over 1.6. In the sample by Hernández-Martínez \& Peña (2009), there are a few candidates with [O III] $\lambda 5007 / \mathrm{H} \alpha$ lower than 1.6 , since other criteria 
were used, such as the non-detection of the central star. Either way, possible low-ionization PNe with faint [O III] $\lambda 5007$ and therefore low stellar effective temperature $\left(T_{*}\right.$ lower than $\left.40 \mathrm{kK}\right)$ were ignored.

As already reported for the $11 \mathrm{PNe}$ studied by Hernández-Martínez et al. (2009), here we again find an old population of PNe with $12+\log (\mathrm{O} / \mathrm{H})<8$ and $12+\log$ $(\mathrm{Ar} / \mathrm{H})<5.7$ (see Figs. 3 and 6 ). These old objects are PN 2, PN 4, PN 8, PN 10, PN 11, PN 13, PN 18, PN 19, PN 21, and $\mathrm{PN} 17$, representing half of the total sample. Seven PNe show $12+\log (\mathrm{O} / \mathrm{H})$ greater than 8.0 and $12+\log (\mathrm{Ar} / \mathrm{H})$ over 5.7, and these young PNe are PN 5, PN 6, PN 7, PN 12, PN 14, PN 16, PN 23, and PN 20. (It is worth noticing that PN 6 is marginally young as its $12+\log (\mathrm{Ar} / \mathrm{H})$ is 5.71.) In Fig. 12 we show the spatial distribution of the two populations in the galaxy. It is observed than young PNe tend to lie in the central zone well inside the optical bar, while the old objects are more widely distributed. The same is true if we plot the PNe velocities (relative to the system) against the distance along the long axis of the stellar spheroid, presented in Fig. 13 (adapted from Flores-Durán et al. 2014); although no preference in velocity is found, the old population is distributed at both sides of the centre, while the young $\mathrm{PNe}$ are nearer the central region in the optical bar. We notice that PN 6 could be marginally an old object.

\section{Summary}

The PN sample analysed here (representing $\sim 84 \%$ of the total sample detected in this galaxy) is biased towards nebulae with very hot central stars. Such a bias probably occurs also in the PN samples of many other external galaxies due to the way PN candidates are selected.

From comparison with stellar evolution models by Karakas (2010) and Fishlock et al. (2014) of the N/O abundance ratio, our PNe should have had initial masses lower than $4 M_{\odot}$, although if the comparison is made with $\mathrm{Ne} / \mathrm{H}$ vs. $\mathrm{O} / \mathrm{H}$ abundances, the initial masses should have been lower than $2 M_{\odot}$. It appears that the models of stars of 2-3 $M_{\odot}$ by Karakas (2010) and Fishlock et al. (2014) are producing too much ${ }^{22} \mathrm{Ne}$ in the stellar surface at the end of the AGB. On the other hand, the comparison with ATON models by Ventura et al. (2013, 2014a,b) - which differ from the previous models in the treatment of convection and on the assumptions concerning the overshoot of the convective core during the core H-burning phase - leads to reasonable agreement between the observed and predicted ratios of $\mathrm{N} / \mathrm{O}$ and $\mathrm{Ne} / \mathrm{H}$ if more massive stars are about $4 M_{\odot}$. So far, none of the models reproduce the large $\mathrm{He}$ abundances found in many $\mathrm{PNe}$ of NGC 6822.

The Type I PNe were produced by stars of any metallicity (not necessarily the richer ones), and their initial masses were between 3 and $4 M_{\odot}$. These objects show the highest effective temperatures.

The PNe in NGC 6822 span in metallicity from very poor objects $(12+\log (\mathrm{Ar} / \mathrm{H}) \leq 5.7)$ to nebulae showing the same metallicity as the $\mathrm{H}$ II regions $(12+\log (\mathrm{Ar} / \mathrm{H}) \sim 5.8)$. The poorer objects are more widely spread in the galaxy, while the young ones lie in or very near the optical bar.

Acknowledgements. This work is based on observations with the Gran Telescopio Canarias (GTC), installed in the Spanish Observatorio del Roque de los Muchachos of the Instituto de Astrofísica de Canarias on the island of La Palma. This work has been funded by the Spanish Ministry of Economy and Competitiveness (MINECO) under the grant AYA2011-22614 and also received partial support from the DGAPA-UNAM, Mexico under grant PAPIIT IN109614. We thank the referee, G. Stasińska, for her valuable comments that helped to improve the quality of the paper. JGR acknowledges support from the Severo Ochoa excellence programme (SEV-2011-0187) postdoctoral fellowship. We are very grateful to Dr. P. Ventura for providing the tables with the surface abundances of his AGB models, and to Dr. C. Morisset for his invaluable help and comments related to the $3 \mathrm{Mdb}$. We also are grateful to A. Karakas from providing the detailed numbers of the models by Fishlock et al. (2014). We acknowledge fruitful discussions with D. A. García-Hernández, A. Karakas, and M. Lugaro.

\section{References}

Allen, C., Carigi, L., \& Peimbert, M. 1998, ApJ, 494, 247

Battinelli, P., Demers, S., \& Kunkel, W. E. 2006, A\&A, 451, 905

Bhatia, A. K., \& Doschek, G. A. 1993, At. Data Nucl. Data Tables, 55, 315

Blöcker, T. 1995, A\&A, 297, 797

Butler, K., \& Zeippen, C. J. 1989, A\&A, 208, 337

Canuto, V. M., \& Mazzitelli, I. 1991, ApJ, 370, 295

Carigi, L., Colin, P., \& Peimbert, M. 2006, ApJ, 644, 924

Ciardullo, R., Feldmeier, J. J., Jacoby, G. H., et al. 2002, ApJ, 577, 31

Corradi, R. L. M., García-Rojas, J., Jones, D., \& Rodríguez-Gil, P. 2015a, ApJ, 803, 99

Corradi, R. L. M., Kwitter, K. B., Balick, B., Henry, R. B. C, \& Hensley, K. 2015b, ApJ, 807, 181

Dance, M., Palay, E., Nahar, S. N., \& Pradhan, A. K. 2013, MNRAS, 435, 1576

de Blok, W. J. G., \& Walter, F. 2000, ApJ, 537, L95

de Blok, W. J. G., \& Walter, F. 2006, AJ, 131, 343

Delgado-Inglada, G., Morisset, C., \& Stasińska, G. 2014, MNRAS, 440, 536

Delgado-Inglada, G., Rodríguez, M., Peimbert, M., Stasińska, G., \& Morisset, C. 2015, MNRAS, 449, 1797

Dell'Agli, F., Ventura, P., Schneider, R., et al. 2015, MNRAS, 447, 2992

Demers, S., Battinelli, P., \& Kunkel, W. E. 2006, ApJ, 636, L85

Esteban, C., Bresolin, F., Peimbert, M., et al. 2009, ApJ, 700, 654

Fishlock, C. K., Karakas, A. I., Lugaro, M., \& Yong, D. 2014, ApJ, 797, 44

Flores-Durán, S., Peña, M., Hernández-Martínez, L. et al. 2014, A\&A, 568, A82

Froese Fischer, C., \& Tachiev, G. 2004, At. Data Nucl. Data Tables, 87, 1

Galavís, M. E., Mendoza, C., \& Zeippen, C. J. 1995, A\&AS, 111, 347

Galavís, M. E., Mendoza, C., \& Zeippen, C. J. 1997, A\&AS, 123, 159

García-Hernández, D. A., \& Górny, S. K. 2014, A\&A, 567, A12

García-Rojas, J., \& Esteban, C. 2007, ApJ, 670, 457

Gieren, W., Pietrzyńki, G., Nalewajko, K., et al. 2006, ApJ, 647, 1056

Hernández-Martínez, L., \& Peña, M. 2009, A\&A, 495, 447

Hernández-Martínez, L., Peña, M., Carigi, L., \& García-Rojas, J. 2009, A\&A, 505,102

Hernández-Martínez , L., Carigi, L., Peña, M., \& Peimbert, M. 2011, A\&A, 535, A118

Henry, R. B. C. 1989, MNRAS, 241, 453

Henry, R. B. C., Kwitter, K. B., \& Balick, B. 2004, AJ, 127, 2284

Hwang, N., Soo Park, H., Gyong Lee, M., et al. 2014, ApJ, 783, 49

Izotov, Y. I., Stasińska, G., Meynet, G., Guseva, N. G., \& Thuan, T. X. 2006, A\&A, 448, 955

Karakas, A. I. 2010, MNRAS, 403, 1413

Karakas, A. I., \& Lattanzio, J. C. 2003, PASA, 20, 393

Karakas, A. I., van Raai, M. A., Lugaro, M., Sterling, N. C., \& Dinerstein, H. L. 2009, ApJ, 690, 1130

Kaufman, V., \& Sugar, J. 1986, J. Phys. Chem. Ref. Data, 15, 343

Kingsburgh R. L., \& Barlow M. J. 1994, MNRAS, 271, 257

Kisielius, R., Storey, P. J., Ferland, G. J., \& Keenan, F. P. 2009, MNRAS, 397, 903

LaJohn, L., \& Luke, T. M. 1993, Phys. Scr., 47, 542

Leisy, P., \& Dennefeld, M. 2006, A\&A, 456, 451

Letarte, B., Demers, S., Batinelli, P., \& Kunkel, W. E. 2002, AJ, 123, 832

Liu, X.-W. 2012, in Planetary Nebulae: An Eye to the Future, eds. A. Manchado, L. Stanghellini, \& D. Schönberner, IAU Symp. 283, 131

Luridiana, V., Morisset, C., \& Shaw, R. A. 2015, A\&A, 573, A42

Magrini, L., Stanguellini, L., \& Villaver, E. 2009, ApJ, 696, 729

Marigo, P. 2001, A\&A, 370, 194

McLaughlin, B. M., \& Bell, K. L. 2000, J. Phys. B, 33, 597

McNabb, I. A., Fang, X., Liu, X.-W., Bastin, R. J., \& Storey, P. J. 2013, MNRAS, 428, 3443

Mendoza, C. 1983, IAU Symp., 103, 143

Mendoza, C., \& Zeippen, C. J. 1982, MNRAS, 198, 127

Moe, M., \& De Marco, O. 2006, ApJ, 650, 916

Morisset, C., Delgado-Inglada, G., \& Flores-Fajardo, N. 2015, Rev. Mex. Astron. Astrofis., 51, 1093 
Peimbert, A., Peimbert, M., \& Ruiz, M. T. 2005, ApJ, 634, 1056 Peña, M., Stasińska, G., \& Richer, M. G. 2007, A\&A, 476, 745

Peña-Guerrero, M. A., Peimbert, A., \& Peimbert, M. 2012, ApJ, 756, L14

Pignatari, M., Herwig, F., Hirschi, R., et al. 2013, ApJS, submitted [arXiv: 1307.6961]

Podobedova, L. I., Kelleher, D. E., \& Wiese, W. L. 2009, J. Phys. Chem. Ref. Data, 38, 171

Ramsbottom, C. A., \& Bell, K. L. 1997, At. Data Nucl. Data Tables, 66, 1

Richer, M. G., \& McCall, M. 2007, ApJ, 658, 328

Rodríguez-González, A., Hernández-Martínez, L., Esquivel, A., et al. 2015, A\&A, 575, A19

Stasińska, G., Richer, M. G., \& McCall, M. 1998, A\&A, 336, 667

Stasińska, G., Peña, M., Bresolin, F., \& Tsamis, Y. G. 2013, A\&A, 552, A12

Storey, P. J., \& Hummer, D. G. 1995, MNRAS, 272, 41
Storey P. J., \& Zeippen C. J., 2000, MNRAS, 312, 813

Storey, P. J., Sochi, T., \& Badnell, N. R. 2014, A\&A, 441, 3028

Tayal, S. S. 2011, ApJS, 195, 12

Tayal, S. S., \& Gupta, G. P. 1999, ApJ, 526, 544

Tayal, S. S., \& Zatsarinny, O. 2010, ApJS, 188, 32

Vassiliadis, E., \& Wood, P. R. 1994, ApJS, 92, 125

Ventura, P., Di Criscienzo, M., Carini, R., \& D’Antona, F. 2013, MNRAS, 431, 3642

Ventura, P., Di Criscienzo, M., D'Antona, F., et al. 2014a, MNRAS, 437, 3274

Ventura, P., Dell'Agli, F., Schneider, R., et al. 2014b, MNRAS, 439, 977

Ventura, P., Stanghellini, L., Dell'Agli, F., García-Hernández, D. A., \& Criscienzo, M. 2015, MNRAS, 452, 3679

Weldrake, D. T. F., de Blok, W. J. G., \& Walter, F. 2003, MNRAS, 340, 12

Wyder, T. K. 2001, AJ, 122, 2490

Wyder, T. K. 2003, AJ, 125, 3097 


\section{Appendix A: Additional figures and tables}
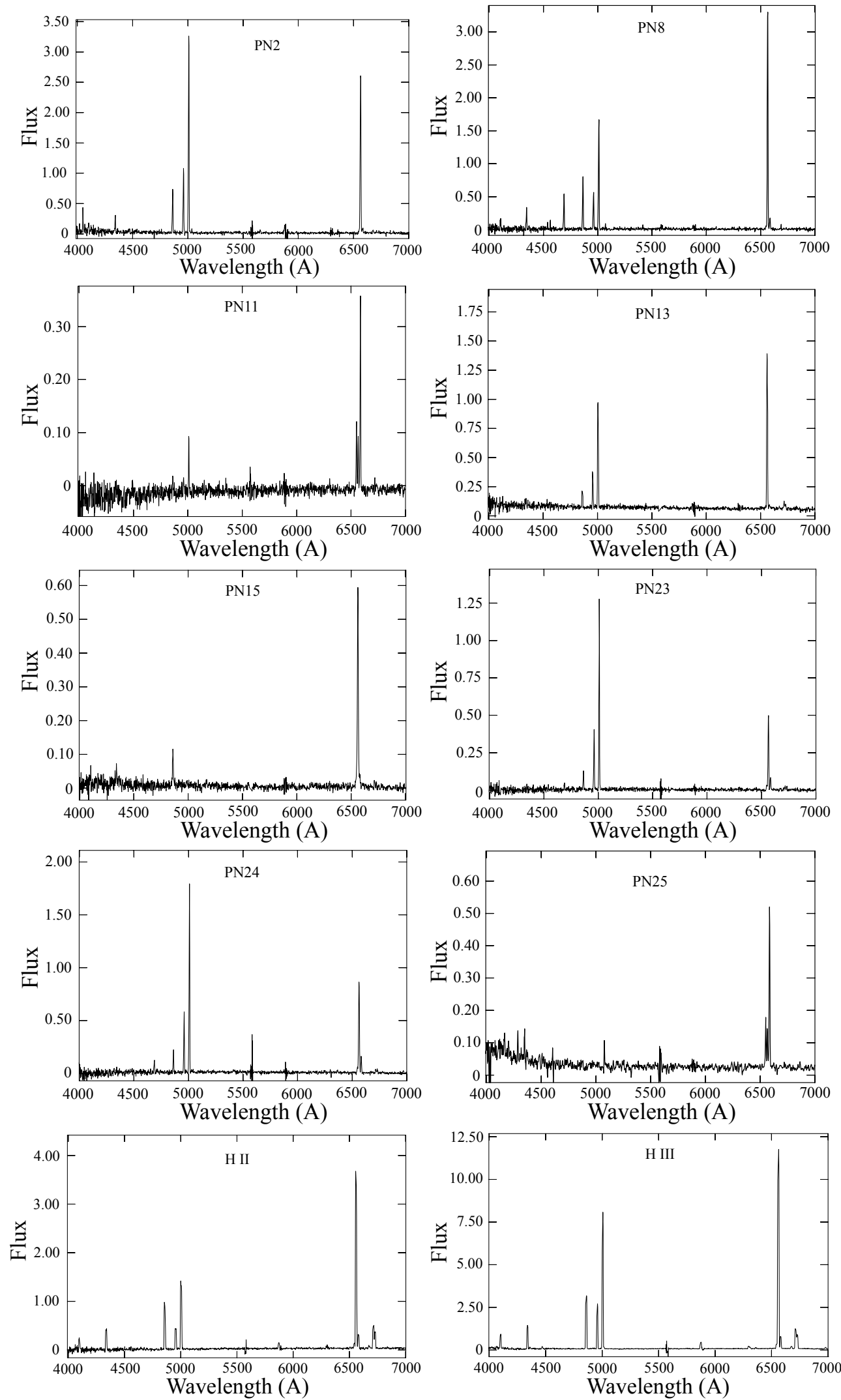

Fig. A.1. Calibrated spectra of PNe and $\mathrm{H}$ II regions observed with GTC. Flux in units of $10^{-16} \mathrm{erg} \mathrm{cm}^{-2} \mathrm{~s}^{-1} \mathrm{~A}^{-1}$. 
Table A.1. Dereddened line intensities from GTC observations.

\begin{tabular}{|c|c|c|c|c|c|c|c|c|c|c|c|c|c|}
\hline \multirow{2}{*}{$\frac{\text { Object }}{\lambda}$} & \multirow[b]{2}{*}{ ion } & \multicolumn{2}{|l|}{ PN 2} & \multicolumn{2}{|l|}{ PN 8} & \multicolumn{2}{|l|}{ PN9 } & \multicolumn{2}{|l|}{ PN 11} & \multicolumn{2}{|l|}{ PN 13} & \multicolumn{2}{|l|}{ PN 15} \\
\hline & & $\mathrm{I} / \mathrm{I}(\mathrm{H} \beta)$ & err & $\mathrm{I} / \mathrm{I}(\mathrm{H} \beta)$ & err & $\mathrm{I} / \mathrm{I}(\mathrm{H} \beta)$ & err & $\mathrm{I} / \mathrm{I}(\mathrm{H} \beta)$ & err & $\mathrm{I} / \mathrm{I}(\mathrm{H} \beta)$ & err & $\mathrm{I} / \mathrm{I}(\mathrm{H} \beta)$ & err \\
\hline 3727 & [O II] & $<2.40 \mathrm{E}-01$ & - & - & - & - & - & - & - & noisy & - & - & - \\
\hline 3869 & [Ne III] & 3.46E-01 & 39 & $<1.00 \mathrm{E}-01$ & - & noisy & - & noisy & - & - & - & - & - \\
\hline 3889 & $\mathrm{H} 8+\mathrm{He} \mathrm{I}$ & - & - & $2.16 \mathrm{E}-01$ & 18 & - & - & noisy & - & noisy & - & - & - \\
\hline 3970 & $\mathrm{H} 7+[\mathrm{Ne} \mathrm{III}]$ & 2.37E-01 & 30 & $1.76 \mathrm{E}-01$ & 20 & - & - & noisy & - & noisy & - & - & - \\
\hline 4102 & $\mathrm{H} \delta$ & $3.08 \mathrm{E}-01$ & 30 & 2.42E-01 & 10 & - & - & noisy & - & 2.35E-01 & 47 & 2.27E-01 & 30 \\
\hline 4340 & $\mathrm{H} \gamma$ & 4.65E-01 & 10 & 4.22E-01 & 8 & 4.43E-01 & 30 & noisy & - & $4.82 \mathrm{E}-01$ & 29 & 4.69E-01 & 11 \\
\hline 4363 & [O III] & $1.30 \mathrm{E}-01$ & 40 & $7.28 \mathrm{E}-02$ & 41 & - & - & noisy & - & 2.44E-01 & 30 & - & - \\
\hline 4471 & He I & $1.77 \mathrm{E}-01$ & 40 & $<3.00 \mathrm{E}-02$ & - & - & - & noisy & - & - & - & - & - \\
\hline 4686 & He II & $<6.00 \mathrm{E}-02$ & - & 7.23E-01 & 5 & - & - & noisy & - & $<1.70 \mathrm{E}-01$ & - & - & - \\
\hline 4861 & $\mathrm{H} \beta$ & $1.00 \mathrm{E}+00$ & 3 & $1.00 \mathrm{E}+00$ & 5 & $1.00 \mathrm{E}+00$ & 5 & $1.00 \mathrm{E}+00$ & 20 & $1.00 \mathrm{E}+00$ & 10 & $1.00 \mathrm{E}+00$ & 3 \\
\hline 4959 & [O III] & $1.43 \mathrm{E}+00$ & 2 & 7.09E-01 & 5 & - & - & $9.92 \mathrm{E}-01$ & 20 & $2.53 \mathrm{E}+00$ & 8 & - & - \\
\hline 5007 & [O III] & $4.49 \mathrm{E}+00$ & 2 & $2.07 \mathrm{E}+00$ & 5 & $2.50 \mathrm{E}-01$ & 40 & $3.09 \mathrm{E}+00$ & 3 & $7.25 \mathrm{E}+00$ & 5 & $<1.00 \mathrm{E}-02$ & - \\
\hline 5412 & He II & - & - & $5.25 \mathrm{E}-02$ & 11 & - & - & - & - & - & - & - & - \\
\hline 5755 & {$[\mathrm{~N} \mathrm{II}]$} & $<2.00 \mathrm{E}-02$ & & $<1.90 \mathrm{E}-02$ & - & - & - & $4.00 \mathrm{E}-01$ & 40 & - & - & - & - \\
\hline 5876 & $\mathrm{HeI}$ & $1.29 \mathrm{E}-01$ & 15 & $6.41 \mathrm{E}-02$ & 25 & - & - & noisy & - & $1.47 \mathrm{E}-01$ & 14 & - & - \\
\hline 6548 & {$[\mathrm{~N} \mathrm{II}]$} & $3.00 \mathrm{E}-02$ & 33 & 4.50E-02 & 22 & - & - & $3.06 \mathrm{E}+00$ & 6 & - & - & $4.00 \mathrm{E}-02$ & 50 \\
\hline 6563 & $\mathrm{H} \alpha$ & $2.86 \mathrm{E}+00$ & 2 & $2.85 \mathrm{E}+00$ & 3 & $2.86 \mathrm{E}+00$ & 1 & $2.86 \mathrm{E}+00$ & 10 & $2.86 \mathrm{E}+00$ & 5 & $2.86 \mathrm{E}+00$ & 3 \\
\hline 6583 & {$[\mathrm{~N} \mathrm{II}]$} & 7.73E-02 & 15 & $1.43 \mathrm{E}-01$ & 6 & $<5.00 \mathrm{E}-02$ & - & $1.06 \mathrm{E}+01$ & 1 & $<5.20 \mathrm{E}-02$ & - & $1.23 \mathrm{E}-01$ & 15 \\
\hline 6678 & $\mathrm{He} \mathrm{I}$ & $5.05 \mathrm{E}-02$ & 20 & $2.70 \mathrm{E}-02$ & 30 & - & - & - & - & - & - & - & - \\
\hline 6716 & {$[\mathrm{~S}$ II $]$} & $<1.50 \mathrm{E}-02$ & - & $6.60 \mathrm{E}-03$ & 30 & $<4.00 \mathrm{E}-02$ & - & 4.52E-01 & 24 & $5.23 \mathrm{E}-02$ & 19 & $4.83 \mathrm{E}-02$ & 19 \\
\hline 6731 & {$[\mathrm{~S} \mathrm{II}]$} & $1.58 \mathrm{E}-02$ & 37 & $1.21 \mathrm{E}-02$ & 49 & $<4.00 \mathrm{E}-02$ & - & 4.38E-01 & 25 & $1.77 \mathrm{E}-02$ & 22 & $4.28 \mathrm{E}-02$ & 21 \\
\hline 7006 & {$[\mathrm{ArV}]$} & - & - & $2.34 \mathrm{E}-02$ & 38 & - & - & - & - & - & - & - & - \\
\hline 7065 & He I & $8.41 \mathrm{E}-02$ & 20 & $1.66 \mathrm{E}-02$ & 54 & - & - & - & - & 7.53E-02 & 20 & - & - \\
\hline 7135 & [Ar III] & $2.34 \mathrm{E}-02$ & 21 & $2.49 \mathrm{E}-02$ & 30 & - & - & $<1.90 \mathrm{E}-01$ & - & $<6.60 \mathrm{E}-02$ & - & - & - \\
\hline 7325 & {$[\mathrm{O} \mathrm{II}]$} & $6.07 \mathrm{E}-02$ & 20 & $2.13 \mathrm{E}-02$ & 42 & $1.00 \mathrm{E}-01$ & 50 & $<3.00 \mathrm{E}-01$ & - & $5.99 \mathrm{E}-02$ & 20 & - & - \\
\hline $\mathrm{F}(\mathrm{H} \beta)^{b}$ & & $5.63 \mathrm{E}-16$ & & $5.94 \mathrm{E}-16$ & & $2.07 \mathrm{E}-16$ & & $2.50 \mathrm{E}-17$ & & $1.15 \mathrm{E}-16$ & & 1.12E-16 & \\
\hline $\mathrm{c}(\mathrm{H} \beta)$ & & 0.58 & & 0.75 & & 1.45 & & 0.126 & & 1.88 & & 1.17 & \\
\hline
\end{tabular}

Notes. Errors in \% . ${ }^{(a)}$ observed flux in $\mathrm{erg}^{-2} \mathrm{~s}^{-1}$.

Table A.1. continued.

\begin{tabular}{|c|c|c|c|c|c|c|c|c|c|c|c|c|c|}
\hline Object & & PN 22 & & PN 23 & & PN 24 & & $\mathrm{PN} 25^{c}$ & & H II & & H III & \\
\hline$\lambda$ & ion & $\mathrm{I} / \mathrm{I}(\mathrm{H} \beta)$ & err & $\mathrm{I} / \mathrm{I}(\mathrm{H} \beta)$ & err & $\mathrm{I} / \mathrm{I}(\mathrm{H} \beta)$ & err & $\mathrm{I} / \mathrm{I}(\mathrm{H} \alpha)$ & err & $\mathrm{I} \mathrm{I} / \mathrm{I}(\mathrm{H} \beta)$ & err & $\mathrm{I} / \mathrm{I}(\mathrm{H} \beta)$ & err \\
\hline 3727 & [O II] & - & - & - & - & - & - & - & - & $5.01 \mathrm{E}+00$ & 10 & $3.76 \mathrm{E}+00$ & 7 \\
\hline 3869 & [Ne III] & - & - & - & - & - & - & - & - & - & - & 1.29E-01 & 12 \\
\hline 3889 & $\mathrm{H} 8+\mathrm{He} \mathrm{I}$ & - & - & - & - & - & - & - & - & - & - & $1.56 \mathrm{E}-01$ & 12 \\
\hline 3970 & $\mathrm{H} 7+[\mathrm{Ne}$ III] & - & - & - & - & - & - & - & - & - & - & $1.88 \mathrm{E}-01$ & 10 \\
\hline 4102 & $\mathrm{H} \delta$ & - & - & - & - & - & - & - & - & $2.45 \mathrm{E}-01$ & 20 & 2.97E-01 & 10 \\
\hline 4340 & $\mathrm{H} \gamma$ & $9.80 \mathrm{E}-01$ & : & $2.77 \mathrm{E}-01$ & : & 4.60E-01 & 20 & - & - & 4.93E-01 & 10 & 4.83E-01 & 10 \\
\hline 4363 & [O III] & - & - & $<2.00 \mathrm{E}-01$ & - & $1.80 \mathrm{E}-01$ & 39 & - & - & $<3.3 \mathrm{E}-02$ & & 2.93E-02 & 20 \\
\hline 4471 & $\mathrm{He} \mathrm{I}$ & - & - & - & - & $<1.00 \mathrm{E}-01$ & - & - & - & - & - & $5.61 \mathrm{E}-02$ & 10 \\
\hline 4686 & He II & - & - & 2.93E-01 & 27 & 7.04E-01 & 15 & - & - & - & - & - & - \\
\hline 4861 & $\mathrm{H} \beta$ & $1.00 \mathrm{E}+00$ & 10 & $1.00 \mathrm{E}+00$ & 10 & $1.00 \mathrm{E}+00$ & 5 & $<2.00 \mathrm{E}-01$ & - & $1.00 \mathrm{E}+00$ & - & $1.00 \mathrm{E}+00$ & 5 \\
\hline 4959 & [O III] $]$ & - & - & $3.43 \mathrm{E}+00$ & 5 & $2.51 \mathrm{E}+00$ & 4 & - & - & 4.83E-01 & 7 & 7.58E-01 & 5 \\
\hline 5007 & [O III] & $<9.00 \mathrm{E}-02$ & - & $1.07 \mathrm{E}+01$ & 4 & $7.77 \mathrm{E}+00$ & 3 & $<2.00 \mathrm{E}-01$ & - & $1.38 \mathrm{E}+00$ & 3 & $2.28 \mathrm{E}+00$ & 3 \\
\hline 5412 & He II & - & - & - & - & $5.00 \mathrm{E}-02$ & 50 & - & - & - & - & - & - \\
\hline 5755 & {$[\mathrm{~N}$ II $]$} & - & - & - & - & $2.00 \mathrm{E}-02$ & 50 & $<1.00 \mathrm{E}-01$ & - & - & - & - & - \\
\hline 5876 & $\mathrm{He} \mathrm{I}$ & - & - & $9.87 \mathrm{E}-02$ & 30 & $<1.00 \mathrm{E}-01$ & - & $<1.00 \mathrm{E}-01$ & - & $1.09 \mathrm{E}-01$ & 10 & 1.03E-01 & 8 \\
\hline 6548 & {$[\mathrm{~N} \mathrm{II}]$} & - & - & $1.50 \mathrm{E}-01$ & 33 & $1.80 \mathrm{E}-01$ & 28 & $1.38 \mathrm{E}+00$ & 10 & 7.39E-02 & 12 & $3.08 \mathrm{E}-02$ & 12 \\
\hline 6563 & $\mathrm{H} \alpha$ & $2.86 \mathrm{E}+00$ & 5 & $2.86 \mathrm{E}+00$ & 5 & $2.87 \mathrm{E}+00$ & 3 & $1.00 \mathrm{E}+00$ & 10 & 2.87E-01 & 3 & $2.89 \mathrm{E}+00$ & 3 \\
\hline 6583 & {$[\mathrm{~N}$ II] } & $3.10 \mathrm{E}-01$ & 23 & $5.32 \mathrm{E}-01$ & 11 & $5.37 \mathrm{E}-01$ & 9 & $4.50 \mathrm{E}+00$ & 3 & $2.24 \mathrm{E}-01$ & 10 & $1.46 \mathrm{E}-01$ & 8 \\
\hline 6678 & $\mathrm{He} \mathrm{I}$ & - & - & - & - & - & - & - & - & $4.19 \mathrm{E}-2$ & 20 & 3.06E-02 & 12 \\
\hline 6716 & {$[\mathrm{~S} \mathrm{II}]$} & $5.80 \mathrm{E}-01$ & 21 & $1.67 \mathrm{E}-01$ & 20 & $1.06 \mathrm{E}-01$ & 19 & $1.10 \mathrm{E}-01$ & 20 & $3.58 \mathrm{E}-01$ & 10 & $2.79 \mathrm{E}-01$ & 8 \\
\hline 6731 & [S II] & 5.10E-01 & 20 & $1.27 \mathrm{E}-01$ & 24 & 8.69E-02 & 23 & $8.10 \mathrm{E}-02$ & 30 & 2.42E-01 & 12 & 2.05E-01 & 8 \\
\hline 7006 & {$[\mathrm{Ar} \mathrm{V}]$} & - & - & - & - & - & - & - & - & - & - & - & - \\
\hline 7065 & $\mathrm{He} \mathrm{I}$ & - & - & - & - & - & - & $4.55 \mathrm{E}-02$ & 44 & $9.75 \mathrm{E}-02$ & 19 & 8.10E-02 & 10 \\
\hline 7135 & [Ar III] & - & - & 8.82E-02 & 29 & $9.34 \mathrm{E}-02$ & 11 & - & - & 7.92E-02 & 20 & 8.19E-02 & 10 \\
\hline 7325 & {$[\mathrm{O} \mathrm{II}]$} & $4.00 \mathrm{E}-01$ & $:$ & $9.48 \mathrm{E}-02$ & 32 & - & - & - & - & $1.36 \mathrm{E}-01$ & 25 & $9.11 \mathrm{E}-02$ & 10 \\
\hline $\mathrm{F}(\mathrm{H} \beta)^{a}$ & & $8.39 \mathrm{E}-17$ & & $8.12 \mathrm{E}-17$ & & $1.45 \mathrm{E}-16$ & & $9.00 \mathrm{E}-17$ & & $1.14 \mathrm{E}-15^{c}$ & & $3.85 \mathrm{E}-15^{c}$ & \\
\hline $\mathrm{c}(\mathrm{H} \beta)$ & & 0.61 & & 0.79 & & 0.89 & & $>0.8$ & & 0.39 & & 0.44 & \\
\hline
\end{tabular}

Notes. Errors in \%. Colons indicate errors greater than 50\%. ${ }^{(a)}$ Observed $\mathrm{H} \beta$ flux in $\mathrm{erg} \mathrm{cm}^{-2} \mathrm{~s}^{-1}$. ${ }^{(b)}$ For PN 25 the reported fluxes are relative to $\mathrm{H} \alpha . \mathrm{F}(\mathrm{H} \alpha)$ is given. ${ }^{(c)}$ Extended $\mathrm{H}$ II region. Partial $\mathrm{H} \beta$ flux in a slit of $3 \times 1.5 \operatorname{arcsec}^{2}$. 
Table A.2. Ionic abundances ${ }^{a}$ in NGC 6822 from VLT (11 PNe and one H II region) and CFH (PN 17 and PN 20) data and for H V and H X ${ }^{b}$

\begin{tabular}{|c|c|c|c|c|c|c|c|c|}
\hline Object & PN 4 & PN 5 & PN 6 & PN 7 & PN 10 & PN 12 & PN 14 & PN 16 \\
\hline $\begin{array}{l}T_{\mathrm{e}}([\mathrm{O} \mathrm{III}])(\mathrm{K}) \\
T_{\mathrm{e}}([\mathrm{N} \mathrm{II}])(\mathrm{K}) \\
n_{\mathrm{e}}([\mathrm{S} \mathrm{II}])\left(\mathrm{cm}^{-3}\right) \\
n_{\mathrm{e}}([\mathrm{Ar} \mathrm{IV}])\left(\mathrm{cm}^{-3}\right)\end{array}$ & $\begin{array}{c}17950 \pm 1250 \\
14200 \pm 2650 \\
2450_{-1000}^{+1800} \\
1900: \\
\end{array}$ & $\begin{array}{c}<24600 \\
15350 \pm 1500 \\
700_{-450}^{+1100} \\
- \\
\end{array}$ & $\begin{array}{c}12500 \pm 750 \\
11600 \pm 3100 \\
3700_{-2150}^{+5200} \\
- \\
\end{array}$ & $\begin{array}{c}13270 \pm 2400 \\
- \\
2300_{-1700}^{+7000} \\
- \\
\end{array}$ & $\begin{array}{c}18200 \pm 1150 \\
- \\
1000 \text { (adopted) } \\
- \\
\end{array}$ & $\begin{array}{c}12900 \pm 650 \\
- \\
2000 \text { (adopted) } \\
1700_{-1250}^{+4600} \\
\end{array}$ & $\begin{array}{c}17900 \pm 1000 \\
20500 \pm 2500 \\
4500_{-2900}^{+8100} \\
11500: \\
\end{array}$ & $\begin{array}{c}14200 \pm 700 \\
- \\
900_{-60}^{+1650} \\
4500_{-2800}^{+7500} \\
\end{array}$ \\
\hline Ion & \multicolumn{8}{|c|}{$12+\log \left(\mathrm{X}^{+i} / \mathrm{H}^{+}\right)$} \\
\hline $\begin{array}{l}\mathrm{He}^{+} \\
\mathrm{He}^{++} \\
\mathrm{N}^{+} \\
\mathrm{O}^{+} \\
\mathrm{O}^{++} \\
\mathrm{Ne}^{++} \\
\mathrm{Ne}^{+4} \\
\mathrm{~S}^{+} \\
\mathrm{S}^{++} \\
\mathrm{Ar}^{++} \\
\mathrm{Ar}^{+3} \\
\mathrm{Ar}^{+4}\end{array}$ & $\begin{array}{c}10.81 \pm 0.04 \\
10.29 \pm 0.04 \\
6.52_{-0.15}^{+0.18} \\
6.85_{-0.24}^{+0.27} \\
7.68 \pm 0.07 \\
6.93 \pm 0.08 \\
6.52 \pm 0.16 \\
5.20_{-0.16}^{+0.19} \\
5.89 \pm 0.10 \\
5.31 \pm 0.07 \\
5.22 \pm 0.09 \\
4.50_{-0.17}^{+0.13} \\
\end{array}$ & $\begin{array}{c}10.80 \pm 0.16 \\
10.66 \pm 0.05 \\
7.40 \pm 0.09 \\
7.05 \pm 0.24 \\
7.70 \pm 0.11 \\
- \\
- \\
5.79 \pm 0.10 \\
6.21_{-0.25}^{+0.20} \\
5.64 \pm 0.11 \\
- \\
- \\
\end{array}$ & $\begin{array}{c}10.98 \pm 0.04 \\
8.93_{-0.25}^{+0.16} \\
6.39_{-0.25}^{+0.32} \\
7.15: \\
8.11 \pm 0.07 \\
7.34 \pm 0.08 \\
- \\
5.16: \\
5.91_{-0.21}^{+0.16} \\
5.55 \pm 0.06 \\
5.23 \pm 0.17 \\
- \\
\end{array}$ & $\begin{array}{c}11.09 \pm 0.10 \\
<9.93 \\
6.48_{-0.13}^{+0.22} \\
7.87: \\
8.01_{-0.17}^{+0.23} \\
- \\
- \\
4.85_{-0.24}^{+0.28} \\
5.88 \pm 0.29 \\
5.79 \pm 0.19 \\
- \\
- \\
\end{array}$ & $\begin{array}{c}11.15 \pm 0.04 \\
<9.36 \\
5.46 \pm 0.12 \\
6.74 \pm 0.13 \\
7.47 \pm 0.06 \\
6.65 \pm 0.08 \\
- \\
- \\
- \\
4.88_{-0.15}^{+0.12} \\
- \\
- \\
\end{array}$ & $\begin{array}{c}10.91 \pm 0.06 \\
10.30 \pm 0.06 \\
5.81 \pm 0.09 \\
6.53_{-0.19}^{+0.24} \\
8.20 \pm 0.08 \\
7.36 \pm 0.09 \\
- \\
4.38_{-0.23}^{+0.26} \\
6.15 \pm 0.12 \\
5.62 \pm 0.09 \\
5.63 \pm 0.11 \\
- \\
\end{array}$ & $\begin{array}{c}10.72 \pm 0.07 \\
10.72 \pm 0.02 \\
7.31_{-0.04}^{+0.07} \\
6.94_{-0.08}^{+0.11} \\
7.87 \pm 0.05 \\
- \\
- \\
4.86_{-0.16}^{+0.25} \\
5.56_{-0.25}^{+0.16} \\
5.39 \pm 0.09 \\
5.32 \pm 0.09 \\
5.13_{-0.13}^{+0.10} \\
\end{array}$ & $\begin{array}{c}11.03 \pm 0.05 \\
10.42 \pm 0.05 \\
6.21 \pm 0.05 \\
6.24 \pm 0.13 \\
8.15 \pm 0.05 \\
7.41 \pm 0.07 \\
- \\
4.67_{-0.09}^{+0.11} \\
5.86_{-0.15}^{+0.12} \\
5.41 \pm 0.08 \\
5.67 \pm 0.08 \\
- \\
\end{array}$ \\
\hline Object & PN 18 & PN 19 & PN 21 & H II 15 & PN 17 & PN 20 & HV & $\mathrm{HX}$ \\
\hline $\begin{array}{l}T_{\mathrm{e}}([\mathrm{O} \mathrm{III}])(\mathrm{K}) \\
T_{\mathrm{e}}([\mathrm{O} \mathrm{II}])(\mathrm{K}) \\
T_{\mathrm{e}}([\mathrm{N} \mathrm{II}])(\mathrm{K}) \\
n_{\mathrm{e}}([\mathrm{S} \mathrm{II}])\left(\mathrm{cm}^{-3}\right) \\
n_{\mathrm{e}}([\mathrm{O} \mathrm{II}])\left(\mathrm{cm}^{-3}\right) \\
n_{\mathrm{e}}([\mathrm{Cl} \mathrm{III}])\left(\mathrm{cm}^{-3}\right) \\
n_{\mathrm{e}}([\mathrm{Ar} \mathrm{IV}])\left(\mathrm{cm}^{-3}\right)\end{array}$ & $\begin{array}{c}18950 \pm 1900 \\
- \\
- \\
1150_{-850}^{+3400} \\
- \\
- \\
-\end{array}$ & $\begin{array}{c}22050 \pm 3300 \\
- \\
- \\
<100 \\
- \\
- \\
-\end{array}$ & $\begin{array}{c}16200 \pm 1200 \\
- \\
- \\
180_{-150}^{+470} \\
- \\
- \\
-\end{array}$ & $\begin{array}{c}12150 \pm 200 \\
- \\
12000 \pm 1800 \\
170_{-100}^{+240} \\
- \\
300_{-210}^{+800} \\
-\end{array}$ & $\begin{array}{c}19300 \pm 3000 \\
- \\
- \\
180_{-120}^{+370} \\
- \\
- \\
-\end{array}$ & $\begin{array}{c}15500 \pm 4000 \\
- \\
- \\
2000 \text { (adopted) } \\
- \\
- \\
-\end{array}$ & $\begin{array}{c}11800 \pm 250 \\
15300 \pm 900 \\
15800 \pm 2850 \\
80_{-50}^{+110} \\
70_{-40}^{+70} \\
600_{-430}^{+1500} \\
-\end{array}$ & $\begin{array}{c}11950 \pm 470 \\
14300 \pm 1100 \\
- \\
<20 \\
50_{-30}^{+70} \\
<20 \\
-\end{array}$ \\
\hline $\begin{array}{l}\mathrm{He}^{+} \\
\mathrm{He}^{++} \\
\mathrm{N}^{+} \\
\mathrm{O}^{+} \\
\mathrm{O}^{++} \\
\mathrm{Ne}^{++} \\
\mathrm{S}^{+} \\
\mathrm{S}^{++} \\
\mathrm{Cl}^{++} \\
\mathrm{Ar}^{++} \\
\mathrm{Ar}^{+3}\end{array}$ & $\begin{array}{c}<10.76 \\
10.82 \pm 0.02 \\
6.40 \pm 0.09 \\
5.72_{-0.14}^{+0.18} \\
7.23_{-0.08}^{+0.10} \\
6.65_{-0.14}^{+0.12} \\
5.09_{-0.17}^{+0.21} \\
- \\
- \\
- \\
- \\
\end{array}$ & $\begin{array}{c}11.09_{-0.26}^{+0.15} \\
10.18 \pm 0.07 \\
6.15_{-0.10}^{+0.14} \\
7.15_{-0.24}^{+0.21} \\
7.19_{-0.09}^{+0.17} \\
- \\
5.76: \\
5.82: \\
- \\
5.30_{-0.15}^{+0.17} \\
- \\
\end{array}$ & $\begin{array}{c}11.11_{-0.09}^{+0.06} \\
9.37_{-0.26}^{+0.16} \\
5.88_{-0.10}^{+0.08} \\
6.50 \pm 0.16 \\
7.74 \pm 0.08 \\
6.96_{-0.10}^{+0.13} \\
5.06 \pm 0.09 \\
<3.61 \\
- \\
5.40 \pm 0.11 \\
- \\
\end{array}$ & $\begin{array}{c}10.90 \pm 0.02 \\
- \\
6.08_{-0.14}^{+0.16} \\
7.58_{-0.21}^{+0.27} \\
7.86 \pm 0.03 \\
7.12 \pm 0.03 \\
5.45_{-0.13}^{+0.15} \\
6.30 \pm 0.06 \\
4.40_{-0.09}^{+0.07} \\
5.76 \pm 0.04 \\
- \\
\end{array}$ & $\begin{array}{c}10.98_{-0.16}^{+0.11} \\
10.54 \pm 0.07 \\
6.06 \pm 0.15 \\
7.28_{-0.19}^{+0.18} \\
7.66_{-0.15}^{+0.18} \\
6.99_{-0.21}^{+0.26} \\
5.78_{-0.11}^{+0.13} \\
- \\
- \\
- \\
- \\
\end{array}$ & $\begin{array}{c}10.91_{-0.07}^{+0.05} \\
9.97_{-0.30}^{+0.17} \\
5.38_{-0.29}^{+0.11} \\
7.06_{-0.17}^{+0.12} \\
7.93 \pm 0.02 \\
7.40_{-0.13}^{+0.10} \\
4.83_{-0.10}^{+0.08} \\
- \\
- \\
5.63 \pm 0.06 \\
- \\
\end{array}$ & $\begin{array}{c}10.92 \pm 0.02 \\
- \\
5.57 \pm 0.15 \\
6.87_{-0.20}^{+0.25} \\
8.04 \pm 0.03 \\
7.30 \pm 0.04 \\
5.09_{-0.12}^{+0.15} \\
6.34 \pm 0.05 \\
4.43 \pm 0.06 \\
5.76 \pm 0.03 \\
4.80 \pm 0.10 \\
\end{array}$ & $\begin{array}{c}10.90 \pm 0.04 \\
- \\
5.80 \pm 0.08 \\
7.14 \pm 0.13 \\
7.94 \pm 0.06 \\
7.18 \pm 0.07 \\
5.29_{-0.08}^{+0.11} \\
6.35 \pm 0.10 \\
4.38_{-0.15}^{+0.11} \\
5.75 \pm 0.05 \\
- \\
\end{array}$ \\
\hline
\end{tabular}

Notes. Abundances in $12+\log \left(\mathrm{X}^{+i} / \mathrm{H}^{+}\right) . \mathrm{HV}$ and $\mathrm{HX}$ line intensities were adopted from Peimbert et al. (2005). Colons indicate errors larger than a factor of two.

Table A.3. Total abundances and $L(\mathrm{H} \beta)$ for PNe from VLT and CFH data and for H V and H X.

\begin{tabular}{|c|c|c|c|c|c|c|c|c|}
\hline Elem/object & PN 4 & PN 5 & PN 6 & PN 7 & PN 10 & PN 12 & PN 14 & PN 16 \\
\hline $\mathrm{He}$ & $10.92 \pm 0.04$ & $11.03 \pm 0.10$ & $10.99 \pm 0.04$ & $11.09 \pm 0.08$ & $11.16 \pm 0.03$ & $11.01 \pm 0.05$ & $11.02 \pm 0.04$ & $11.13 \pm 0.04$ \\
\hline $\mathrm{N}$ & $7.43 \pm 0.09$ & $8.21_{-0.12}^{+0.17}$ & $7.55 \pm 0.18$ & $6.89_{-0.16}^{+0.18}$ & $6.37_{-0.15}^{+0.13}$ & $7.50 \pm 0.17$ & $8.39 \pm 0.12$ & $8.13 \pm 0.12$ \\
\hline $\mathrm{O}$ & $7.81 \pm 0.10$ & $7.94 \pm 0.13$ & $8.16_{-0.08}^{+0.13}$ & $8.27_{-0.21}^{+0.30}$ & $7.55 \pm 0.07$ & $8.26 \pm 0.08$ & $8.12 \pm 0.04$ & $8.21 \pm 0.05$ \\
\hline $\mathrm{Ne}$ & $7.00 \pm 0.08$ & - & 7.65: & -0.21 & $6.75_{-0.11}^{+0.20}$ & $7.42 \pm 0.08$ & - & $7.47 \pm 0.07$ \\
\hline$S$ & $6.18 \pm 0.10$ & $6.59_{-0.19}^{+0.16}$ & $6.17 \pm 0.17$ & $5.92_{-0.25}^{+0.30}$ & - & $6.57 \pm 0.12$ & $6.01_{-0.22}^{+0.17}$ & $6.37 \pm 0.14$ \\
\hline $\mathrm{Ar}$ & $5.52 \pm 0.08$ & $5.90 \pm 0.12$ & $5.71 \pm 0.09$ & $5.84 \pm 0.16$ & $5.01_{-0.16}^{+0.11}$ & $5.94 \pm 0.10$ & $5.74 \pm 0.10$ & $5.70 \pm 0.08$ \\
\hline$L(\mathrm{H} \beta) / L_{\odot}$ & 33.92 & 3.27 & 20.16 & 7.32 & 26.95 & 3.18 & 15.98 & 5.73 \\
\hline Elem/object & PN 18 & PN 19 & PN 21 & H II 15 & PN 17 & PN 20 & $\mathrm{HV}$ & $\mathrm{HX}$ \\
\hline $\mathrm{He}$ & $11.09 \pm 0.09$ & $11.14_{-0.21}^{+0.14}$ & $11.11_{-0.09}^{+0.05}$ & $10.95 \pm 0.03$ & $11.12_{-0.11}^{+0.09}$ & $10.96_{-0.07}^{+0.06}$ & $10.94 \pm 0.02$ & $10.95 \pm 0.03$ \\
\hline $\mathrm{N}$ & $8.04_{-0.12}^{+0.14}$ & $6.50_{-0.11}^{+0.33}$ & $7.26_{-0.13}^{+0.179}$ & $6.54 \pm 0.04$ & $6.62_{-0.14}^{+0.111}$ & $6.33_{-0.33}^{+0.21}$ & $6.72 \pm 0.05$ & $6.63 \pm 0.06$ \\
\hline $\mathrm{O}$ & $7.48_{-0.11}^{+0.16}$ & $7.50_{-0.14}^{+0.11}$ & $7.77 \pm 0.08$ & $8.05_{-007}^{+0.11}$ & $7.89 \pm 0.16$ & $8.01 \pm 0.03$ & $8.07 \pm 0.03$ & $8.00 \pm 0.05$ \\
\hline $\mathrm{Ne}$ & $6.88_{-0.16}^{+0.119}$ & - & $7.00_{-0.16}^{+0.21}$ & $7.18 \pm 0.04$ & $7.07_{-0.21}^{+0.24}$ & $7.42_{-0.12}^{+0.11}$ & $7.31 \pm 0.04$ & $7.21 \pm 0.07$ \\
\hline S & $6.88 \pm 0.13$ & 6.10: & $6.63_{-0.15}^{+0.19}$ & $6.39 \pm 0.05$ & $6.56_{-0.11}^{+0.11}$ & $6.04_{-0.16}^{+0.12}$ & $6.63 \pm 0.07$ & $6.51 \pm 0.10$ \\
\hline $\mathrm{Cl}$ & - & - & - & $4.50 \pm 0.09$ & - & -- & $4.86_{-0.08}^{+0.10}$ & $4.60 \pm 0.14$ \\
\hline $\mathrm{Ar}$ & - & $5.34 \pm 0.19$ & $5.60_{-0.12}^{+0.09}$ & $5.79 \pm 0.04$ & - & $5.80 \pm 0.06$ & $5.81 \pm 0.03$ & $5.84 \pm 0.06$ \\
\hline$L(\mathrm{H} \beta) / L_{\odot}$ & 0.57 & 1.23 & 0.76 & 308.67 & - & - & - & - \\
\hline
\end{tabular}

Notes. Abundances in $12+\log (\mathrm{X} / \mathrm{H})$. Colons indicate errors larger than a factor of two. 